\title{
Einstein relation for reversible diffusions in random environment
}

\author{
N. Gantert* $\quad$ P. Mathieu ${ }^{* *} \quad$ A. Piatnitski ${ }^{\S}$
}

July 27,2018

\begin{abstract}
We consider reversible diffusions in random environment and prove the Einstein relation for this model. It says that the derivative at 0 of the effective velocity under an additional local drift equals the diffusivity of the model without drift. The Einstein relation is conjectured to hold for a variety of models but it is proved insofar only in particular cases. Our proof makes use of homogenization arguments, the Girsanov transform, and a refinement of the regeneration times introduced in [25].
\end{abstract}

\section{Introduction}

The present paper deals with diffusions in a random stationary environment, a model for the dynamics of particles in a disordered medium at thermal equilibrium. This subject has been the object of intense research over the past thirty years. In spite of many recent progresses, see [27] for instance, many questions regarding the long time behavior of these processes, such as laws of large numbers or central limit theorems, are still challenging open problems.

We shall only be concerned with reversible dynamics. In this context the idea of the environment seen from the particle, as discussed in [13] or [4], provides a powerful tool to adapt the 'corrector approach' from homogenization theory and eventually prove invariance principles. One then shows that the trajectory of a particle evolving in such an environment, in a large time scale, behaves like a Brownian motion with mean square displacement proportional to time, the proportionality being expressed by the asymptotic covariance or effective diffusivity matrix $\Sigma$. A good understanding of $\Sigma$ is thus of primary interest.

¿From reversibility follows a variational formula for the effective diffusivity, see [18] and [12] for the discrete and continuous cases, respectively. Many works in PDE or theoretical physics address the question of estimating the effective diffusivity. See [7] or [8] for instance. Here we provide a completely different interpretation of $\Sigma$ as the so-called mobility.

\footnotetext{
*Technische Universität München, Fachbereich Mathematik und Informatik, Boltzmannstr. 3, 85748 Garching, GERMANY.gantert@ma.tum.de

**Université de Provence, CMI, 39 rue Joliot-Curie, 13013 Marseille, FRANCE. pierre.mathieu@ cmi.univ-mrs.fr

${ }^{\S}$ Lebedev Physical Institute of Russian Academy of Sciences and Narvik Institute of Technology, P. O. Box 385, N-8505 Narvik, NORWAY. andrey@ sci.lebedev.ru
} 
In the series of papers A. Einstein devoted to Brownian motion at the beginning of past century, see [5], along with the diffusivity matrix, the great physicist introduced another important quantity called the mobility. The mobility measures the response of the diffusing particle to a constant exterior force. Let us consider the perturbed process obtained by imposing a constant drift of strength $\lambda$ in some fixed direction. One would expect the perturbed process to satisfy a law of large numbers with effective drift $\ell(\lambda)$. The mobility can then be interpreted as the derivative of $\ell(\lambda)$ as $\lambda$ tends to 0 . Einstein claimed that the mobility and the diffusivity of a particle coincide.

This 'diffusivity vs mobility' relation played a central role in Einstein's theory of molecular diffusivity because it was amenable to experimental verifications and eventually lead to evidences that matter is molecular. Since then, Einstein's relation opened the way to important developments both in experimental physics, with J. Perrin's Nobel prize, and theoretical physics, with connections to the Fluctuation Dissipation Theorem or the Green-Kubo relation. Authors in these fields usually assume the Einstein relation, on the basis on heuristics, without being actually able to prove it, see [8] for instance. Indeed, only very few rigorous papers investigate the mathematical contents of the Einstein relation and finding a general strategy for proving it remained an open mathematical problem for years. Note that the mere existence of the mobility i.e. the fact that $\ell(\lambda)$ has a derivative at $\lambda=0$ is far from obvious.

In the case of periodic environments the Einstein relation can be easily checked by direct computations which are mostly reduced to differentiating the perturbed cell problem on the torus. More generally, if the process of the environment seen from the particle has a spectral gap, the Einstein relation can be proved by perturbation theory, see [16].

The first consistent mathematical approach to the Einstein relation for random environments was attempted by J. Lebowitz and H. Rost in [19]. These authors then avoided the difficulty of proving the existence of the mobility by considering exterior forces whose strength vanishes as time goes to infinity in such a way that the perturbed process still has a limit on the diffusive scale. The mobility is then defined as the asymptotic mean displacement of the particle; no derivative is involved and this weak form of the Einstein relation does not tell us anything about the effective drift induced by a constant exterior force. On the other hand, in this weaker form the Einstein relation holds in the general ergodic reversible case. We shall discuss the Lebowitz-Rost theory in more details later, see the end of section 2.4 .

More recently, in [15], T. Komorowski and S. Olla proved the Einstein relation for random walks with random conductances in dimension higher than 3 and with the extra restriction that conductances are only allowed to take two different positive values. Their approach, which is an adaptation of [21] to the RWRE case, is quite different from ours and it is not clear how it can be adapted to more general models, as for instance random walks on percolation clusters, see [2] and [26].

In the present paper, we shall prove the Einstein relation for symmetric diffusions driven by a random environment with bounded potential and short range correlations. A more detailed definition of the model and precise assumptions are given in part 2. As discussed above, the invariance principle, and therefore the existence of the diffusivity, were already known for such models in the eighties. The law of large numbers for the perturbed dynamics, i.e. the existence of $\ell(\lambda)$, is ensured by a more general theorem of L. Shen in [25] on renewal properties of drifted diffusions in random environments. The key issue in proving the Einstein relation is then to show that $\ell(\lambda)$ has a derivative at $\lambda=0$ and to identify this derivative with the effective diffusivity of the non-perturbed diffusion. To this end we compute the mean position of the perturbed diffusion on the critical scale $\lambda^{2} t=1$. This is indeed the scale on which the diffusive and drift components of the perturbed dynamics equilibrate. Thus we 
obtain two different expressions for the mean displacement in terms of either the diffusivity matrix $\Sigma$, see equation (3.1), or in terms of $\ell(\lambda)$, see (5.1). Identifying both expressions gives the Einstein relation.

The proofs combine different ingredients: homogenization arguments and Girsanov transforms, - see Section 3 - PDE estimates and a-priori bounds on hitting times for perturbed diffusions - see Section 4 - and renewal arguments - see Section 5. All these ingredients had already appeared in the literature but, in order to treat the critical scale $\lambda^{2} t=1$, we had to refine many arguments and often introduce alternative strategies as, for instance, with the regeneration times in Section 5.

In Sections 2 - 6, we focus only on smooth environments which allows us to use stochastic differential equations. In Section 7, we relax this smoothness assumption and treat the case of (still bounded) but only measurable environments, relying on Dirichlet form theory.

One might hope that our approach could be adapted to apply to other models of diffusions or random walks in random environments.

\section{Model and statement of the theorem}

\subsection{Diffusions in a random environment}

We shall be dealing with diffusion processes in $\mathbb{R}^{d}$ whose generators are of the form

$$
\mathscr{L}^{\omega} f(x)=\frac{1}{2} e^{2 V^{\omega}(x)} \operatorname{div}\left(e^{-2 V^{\omega}} a^{\omega} \nabla f\right)(x),
$$

where $a^{\omega}$ and $V^{\omega}$ are realizations of a random environment with finite range of dependence.

More precisely, our assumptions are as follows.

Let $(\Omega, \mathscr{A}, \mathbb{Q})$ be a probability space equipped with a group action of $\mathbb{R}^{d}$ that we denote with $(x, \omega) \rightarrow x . \omega$. We also assume that the map $(x, \omega) \rightarrow x . \omega$ is $\left(\mathscr{B}_{d} \times \mathscr{A}, \mathscr{A}\right)$-measurable, where $\mathscr{B}_{d}$ is the Borel $\sigma$-field on $\mathbb{R}^{d}$.

Assumption 1: the action $(x, \omega) \rightarrow x . \omega$ preserves the measure $\mathbb{Q}$ and is ergodic.

Let $V$ be a measurable real-valued function on $\Omega$ and let $\sigma$ be a measurable function on $\Omega$ taking its values in the set of real $d \times d$ symmetric matrices. Define

$$
V^{\omega}(x)=V(x . \omega), \sigma^{\omega}(x)=\sigma(x . \omega) .
$$

We also introduce the notation

$$
a^{\omega}=\left(\sigma^{\omega}\right)^{2} \text { and } b^{\omega}=\frac{1}{2} \operatorname{div} a^{\omega}-a^{\omega} \nabla V^{\omega} .
$$

Observe that both $\sigma^{\omega}$ and $b^{\omega}$ are then stationary fields i.e. $\sigma^{\omega}(x)=\sigma(x . \omega)$ and $b^{\omega}(x)=b(x . \omega)$ for some functions $\sigma$ and $b$.

Assumption 2: for any environment $\omega$, the functions $x \rightarrow V^{\omega}(x)$ and $x \rightarrow \sigma^{\omega}(x)$ are smooth. To avoid triviality, we also assume that at least one of them is not constant.

Assumption 3: $V$ is bounded and $a^{\omega}$ is uniformly elliptic, namely there exists a constant $\kappa$ such that, for all $\omega, x$ and $y$,

$$
\kappa|y|^{2} \leq\left|\sigma^{\omega}(x) y\right|^{2} \leq \kappa^{-1}|y|^{2} .
$$


For a Borel subset $F \subset \mathbb{R}^{d}$, we define the $\sigma$-field

$$
\mathscr{H}_{F}=\sigma\{V(x . \omega), \sigma(x . \omega): x \in F\}
$$

and we assume the following independence condition:

Assumption 4: there exists $R$ such that for any Borel subsets $F$ and $G$ such that $d(F, G)>R$ (where $d(F, G)=\inf \{|x-y|: x \in F, y \in G\}$ is the distance between $F$ and $G)$ then

$$
\mathscr{H}_{F} \text { and } \mathscr{H}_{G} \text { are independent. }
$$

Let $\left(W_{t}: t \geq 0\right)$ be a Brownian motion defined on some probability space $(\mathscr{W}, \mathscr{F}, P)$. We denote expectation with respect to $P$ by $E$. By diffusion in the environment $\omega$ we mean the solution of the stochastic differential equation

$$
d X_{x}^{\omega}(t)=b^{\omega}\left(X_{x}^{\omega}(t)\right) d t+\sigma^{\omega}\left(X_{x}^{\omega}(t)\right) d W_{t} ; X_{x}^{\omega}(0)=x .
$$

Then $X_{x}^{\omega}$ is indeed the Markov process generated by the operator $\mathscr{L}^{\omega}$ in equation (2.1). We shall denote with $P_{x}^{\omega}$ the law of $X^{\omega}$ on the path space $C\left(\mathbb{R}_{+}, \mathbb{R}^{d}\right)$. It is usually referred to as the quenched law of the diffusion in a random environment. We will also need the so-called annealed law:

$$
\mathbb{P}_{x}[A]:=\int d \mathbb{Q}(\omega) \int d P_{x}^{\omega}(w) \mathbf{1}_{A}(\omega, w)
$$

for any measurable subset $A \subset \Omega \times C\left(\mathbb{R}_{+}, \mathbb{R}^{d}\right)$.

Expectation with respect to $P_{x}^{\omega}$ will be denoted with $E_{x}^{\omega}$ and expectation with respect to $\mathbb{P}_{x}$ will be denoted with $\mathbb{E}_{x}$.

We use the notation $X(t)$ for the coordinate process on path space $C\left(\mathbb{R}_{+}, \mathbb{R}^{d}\right)$.

\subsection{Effective diffusivity}

Definition 2.1 Let $\Sigma$ be the effective diffusivity matrix defined by

$$
e \cdot \Sigma e:=\lim _{t \rightarrow+\infty} \frac{1}{t} \mathbb{E}_{0}\left[(e \cdot X(t))^{2}\right]
$$

where $e$ is any vector in $\mathbb{R}^{d}$ and $x \cdot y$ denotes the scalar product of the two vectors $x$ and $y$.

The fact that the limit in (2.6) exists is (almost) a consequence of the Central Limit Theorem for the process $X$ under $\mathbb{P}_{0}$. More is actually known: $X$ satisfies a full invariance principle. Namely: for almost any realization of the environment $\omega$, the laws of the sequence of rescaled processes $\left(X^{\varepsilon}(t)=\varepsilon X\left(t / \varepsilon^{2}\right) ; t \geq 0\right)$ under $P_{0}^{\omega}$ weakly converge as $\varepsilon$ goes to 0 to the law of a Brownian motion with covariance matrix $\Sigma$. References on this Theorem include [4], [13], [17], [22], [23] among others. The convergence of the variance of the process to $\Sigma$ is explicitly stated in [4] formula (2.44).

The invariance principle also has a PDE counterpart in terms of homogenization theory, see for instance the book [12]. The generator of the process $X^{\varepsilon}$ under $P_{x}^{\omega}$ is the rescaled elliptic operator with rapidly oscillating coefficients

$$
\frac{1}{2} a^{\omega}\left(\frac{\dot{\varepsilon}}{\varepsilon}\right) \Delta+\frac{1}{2 \varepsilon} b^{\omega}\left(\frac{\dot{\varepsilon}}{\varepsilon}\right) \nabla \text {. }
$$


Its limit, in the sense of homogenization theory, is the elliptic operator with constant coefficient

$$
\frac{1}{2} \operatorname{div}(\Sigma \nabla)
$$

where $\Sigma$ is the same matrix as in (2.6).

The effective diffusivity $\Sigma$ is a symmetric matrix. As a consequence of Assumption 1 on ergodicity, $\Sigma$ is deterministic (i.e. $\Sigma$ does not depend on $\omega$ ). Furthermore, due to the ellipticity Assumption $3, \Sigma$ is also known to be positive definite.

In general, there is no simple expression of $\Sigma$ in terms of $\sigma$ or $V$. (For instance, $\Sigma$ is by no means the average of $\sigma^{2}$ !) The proof of the homogenization theorem actually provides an expression of $\Sigma$ as a function of the solution of a Poisson equation - the so-called corrector approach. Since the operator $\mathscr{L}^{\omega}$ is self-adjoint with respect to the measure $e^{-2 V^{\omega}(x)} d x$, there is also a variational formulation of the Poisson equation and therefore a variational formula for $\Sigma$ itself. We will not need it in this paper. Our main Theorem actually gives a quite different interpretation of $\Sigma$ as the mobility of $X^{\omega}$, see below.

\subsection{Perturbed diffusions}

We shall now consider perturbations of the process $X$ obtained by inserting a local drift in equation (2.4).

We use the following notation. Let $e_{1}$ be a non-zero vector with $\left|e_{1}\right|=1$ and $\lambda>0$. We define $\hat{\lambda}$ to be the vector $\hat{\lambda}=\lambda e_{1}$. We think of $e_{1}$ as being fixed while $\lambda$ is due to tend to 0 . We assume throughout the whole paper that $\lambda \leq 1$.

Let us consider the perturbed stochastic differential equation:

$$
d X_{x}^{\lambda, \omega}(t)=b^{\omega}\left(X_{x}^{\lambda, \omega}(t)\right) d t+a^{\omega}\left(X_{x}^{\lambda, \omega}(t)\right) \hat{\lambda} d t+\sigma^{\omega}\left(X_{x}^{\lambda, \omega}(t)\right) d W_{t} ; X_{x}^{\lambda, \omega}(0)=x .
$$

The process $X_{x}^{\lambda, \omega}$ is now a Markov process with generator

$$
\begin{aligned}
\mathscr{L}^{\lambda, \omega} f(x) & =\frac{1}{2} e^{2 V^{\omega}(x)} \operatorname{div}\left(e^{-2 V^{\omega}} a^{\omega} \nabla f\right)(x)+a^{\omega}(x) \hat{\lambda} \cdot \nabla f(x) \\
& =\frac{1}{2} e^{2 V^{\lambda, \omega}(x)} \operatorname{div}\left(e^{-2 V^{\lambda, \omega}} a^{\omega} \nabla f\right)(x),
\end{aligned}
$$

where $V^{\lambda, \omega}(x)=V^{\omega}(x)-\hat{\lambda} \cdot x$. We shall use the notation $P_{x}^{\lambda, \omega}$ for the law of $X_{x}^{\lambda, \omega}, E_{x}^{\lambda, \omega}$ for the corresponding expectation as well as $\mathbb{P}_{x}^{\lambda}$ and $\mathbb{E}_{x}^{\lambda}$ for the annealed probability and expectation defined analogously to (2.5).

Our model is a special case of diffusions with drifts considered by L. Shen in [25] for which the author proved a law of large numbers: for almost any environment $\omega$, the ratio $X(t) / t$ has an almost sure limit under $P_{0}^{\lambda, \omega}$, say $\ell(\lambda)$. The convergence also holds in $L^{1}\left(\mathbb{P}_{0}^{\lambda}\right)$. Moreover $\ell(\lambda)$ is deterministic and $\hat{\lambda} \cdot \ell(\lambda)>0$. Note that the proof strongly relies on the independence property Assumption 4. We thus define the effective velocity:

Definition 2.2 Let $\lambda>0$. Let $\ell(\lambda)$ be the effective drift vector defined by

$$
\ell(\lambda)=\lim _{t \rightarrow+\infty} \frac{1}{t} \mathbb{E}_{0}^{\lambda}[X(t)]
$$

By convention $\ell(0)=0$. 


\subsection{The Einstein relation}

Our main result is the following theorem.

Theorem 2.3 The function $\lambda \rightarrow \ell(\lambda)$ has a derivative at $\lambda=0$ which satisfies

$$
\lim _{\lambda \rightarrow 0} \frac{1}{\lambda} \ell(\lambda)=\Sigma e_{1}
$$

This justifies the following definition.

Definition 2.4 Call mobility in the direction $e_{2}$ the derivative at $\lambda=0$ of the velocity $e_{2} \cdot \ell(\lambda)$.

Theorem 2.3 and Definition 2.4 can be compared with the main result of [19] where the mobility is defined as the mean position of the process $X^{\varepsilon}$ under $\mathbb{P}_{0}^{\varepsilon}$. The authors prove that, as $\varepsilon$ tends to 0 , the law of $X^{\varepsilon}$ under $\mathbb{P}_{0}^{\varepsilon}$ converges to the law of Brownian motion with drift $v$ given by $v=\sum e_{1}$. These results are consequences of the invariance principle under $\mathbb{P}_{0}$ and do not require any information on the asymptotic behaviour of the process under $\mathbb{P}_{0}^{\lambda}$ for a fixed $\lambda$ (and indeed the law of large numbers of L. Shen was not known at the time [19] was written).

\section{Girsanov transforms}

The aim of this section is to establish Proposition 3.1 below. In this part of the paper we only use Assumptions 1, 2, 3.

Proposition 3.1 Let $\alpha \geq 1$. Then

$$
\lim _{\lambda \rightarrow 0 ; t \rightarrow+\infty ; \lambda^{2} t=\alpha} \mathbb{E}_{0}^{\lambda\left[\frac{X(t)}{\lambda t}\right]=\Sigma e_{1} .}
$$

Also

$$
\sup _{\alpha \geq 1} \limsup _{\lambda \rightarrow 0 ; t \rightarrow+\infty ; \lambda^{2} t=\alpha} \mathbb{E}_{0}^{\lambda}\left[\frac{\max _{s \leq t}|X(s)|^{2}}{\lambda^{2} t^{2}}\right]<\infty,
$$

Remark 3.2 Observe that (3.2) directly follows from Lemma 4.5 in the next section of the paper.

Proof of Proposition 3.1 in the case $V=0$

We first prove Proposition 3.1 in the case there is no potential i.e. we start assuming that $V=0$.

We use Girsanov transforms pretty much as in [19] i.e. the explicit expression of the RadonNikodym derivative of $P_{0}^{\lambda, \omega}$ with respect to $P_{0}^{\omega}$.

Let us first recall Girsanov transforms, see [24], chapter VIII. Let $X=\left(X_{1}, \ldots, X_{d}\right)$ and $Y=$ $\left(Y_{1}, \ldots, Y_{d}\right)$ be solutions of stochastic differential equations of the form

$$
d X(t)=b(X(t)) d t+\sigma(X(t)) d W_{t} ; X(0)=x
$$


and

$$
d Y(t)=c(Y(t)) d t+\sigma(Y(t)) d W_{t} ; Y(0)=x,
$$

where $\left(W_{t}: t \geq 0\right)$ is a Brownian motion and the coefficients $\sigma, b$ and $c$ are subject to smoothness and ellipticity assumptions as in Assumptions 2 and 3. Let $P^{X}$ and $P^{Y}$ be the laws of the processes $X$ and $Y$ on the path space $C\left(\mathbb{R}_{+}, \mathbb{R}^{d}\right)$. Let $\mathscr{F}_{t}=\sigma\{X(s) ; s \leq t\}$ be the filtration generated by the coordinate process up to time $t$. Then the restriction of $P^{Y}$ to $\mathscr{F}_{t}$ is absolutely continuous with respect to the restriction of $P^{X}$ to $\mathscr{F}_{t}$ and the Radon-Nikodym derivative is given by the Girsanov formula:

$$
E[F(Y([0, t]))]=E\left[F(X([0, t])) e^{M(t)-\frac{1}{2}\langle M\rangle(t)}\right],
$$

for any time $t$, for any bounded continuous function $F$ on $C\left([0, t], \mathbb{R}^{d}\right)$ and where $M$ is the martingale

$$
M(t)=\int_{0}^{t} \phi(X(s)) \cdot d W_{s}
$$

$\langle M\rangle$ is its bracket

$$
\langle M\rangle(t)=\int_{0}^{t}|\phi(X(s))|^{2} d s
$$

and $\phi(x)=\sigma^{-1}(x)(c(x)-b(x))$.

In the next discussion we use the expression "Brownian motion with covariance $\sigma^{2}$ and drift $c$ " to denote any process whose law is the same as the law of $\left(\sigma W_{t}+c t ; t \geq 0\right)$.

If we choose $\sigma$ and $c$ constant and $b=0$ above, then $Y$ is a Brownian motion with covariance $\sigma^{2}$ and drift $c$ and $(X, M)$ is a centered Brownian motion (in dimension $d+1$ ) whose covariance satisfies the following: the covariance of $X$ is $\sigma^{2} ; E[M(t) X(t)]=c t$. Thus the Girsanov formula then has the following corollary: let $Y$ be a Brownian motion with covariance matrix $\sigma^{2}$ and $\operatorname{drift} c$, then

$$
E[F(Y([0, t]))]=E\left[F(X([0, t])) e^{M(t)-\frac{1}{2}\langle M\rangle(t)}\right],
$$

for any time $t$, for any bounded continuous function $F$ on $C\left([0, t], \mathbb{R}^{d}\right)$ and for any random process $M$ such that $(X, M)$ is a centered Brownian motion, $X$ has covariance $\sigma^{2}$ and the covariance of $X$ and $M$ is $E[M(t) X(t)]=c t$.

Applying the Girsanov formula to the processes $X^{\omega}$ and $X^{\lambda, \omega}$, we get that, for any $\omega$,

$$
E\left[F\left(X_{0}^{\lambda, \omega}([0, t])\right)\right]=E\left[F\left(X_{0}^{\omega}([0, t])\right) e^{\lambda \bar{B}(t)-\frac{\lambda^{2}}{2}\langle\bar{B}\rangle(t)}\right]
$$

where $\bar{B}$ is the martingale

$$
\bar{B}(t)=\int_{0}^{t} \sigma^{\omega}\left(X_{0}^{\omega}(s)\right) e_{1} \cdot d W_{s}
$$

and $\langle\bar{B}\rangle$ is its bracket

$$
\langle\bar{B}\rangle(t)=\int_{0}^{t}\left|\sigma^{\omega}\left(X_{0}^{\omega}(s)\right) e_{1}\right|^{2} d s .
$$

In particular, in the range $\lambda^{2} t=\alpha$, we have

$$
E\left[F\left(X_{0}^{\lambda, \omega}([0, t])\right)\right]=E\left[F\left(X_{0}^{\omega}([0, t])\right) e^{\lambda \bar{B}\left(\frac{\alpha}{\lambda^{2}}\right)-\frac{\lambda^{2}}{2}\langle\bar{B}\rangle\left(\frac{\alpha}{\lambda^{2}}\right)}\right] .
$$

We shall need the following easy statement: 
Lemma 3.3 For all $\alpha \geq 1$ and $\mu>1$, we have

$$
\limsup _{\lambda \rightarrow 0 ; t \rightarrow+\infty ; \lambda^{2} t=\alpha} E\left[e^{\mu \lambda \bar{B}\left(\frac{\alpha}{\lambda^{2}}\right)-\frac{\mu \lambda^{2}}{2}\langle\bar{B}\rangle\left(\frac{\alpha}{\lambda^{2}}\right)}\right]<\infty,
$$

uniformly in $\omega$.

Proof of (3.5)

Assumption 3 on the ellipticity of $a^{\omega}$ implies that $\langle\bar{B}\rangle(t) \leq \kappa^{-1} t$. Therefore

$$
\begin{aligned}
& E\left[e^{\mu \lambda \bar{B}\left(\frac{\alpha}{\lambda^{2}}\right)-\frac{\mu \lambda^{2}}{2}\langle\bar{B}\rangle\left(\frac{\alpha}{\lambda^{2}}\right)}\right] \\
\leq & E\left[e^{\mu \lambda \bar{B}\left(\frac{\alpha}{\lambda^{2}}\right)-\frac{\mu^{2} \lambda^{2}}{2}\langle\bar{B}\rangle\left(\frac{\alpha}{\lambda^{2}}\right)}\right] e^{(\mu-1) \frac{\mu \lambda^{2}}{2} \frac{\alpha}{\kappa \lambda^{2}}} \\
= & e^{(\mu-1) \frac{\mu}{2} \frac{\alpha}{\kappa}}
\end{aligned}
$$

and (3.5) is thus proved.

Next we apply a (joint) invariance principle for the process $\left(X_{0}^{\omega, \varepsilon}, \bar{B}^{\varepsilon}\right)$ where $X_{0}^{\omega, \varepsilon}(t)=\varepsilon X_{0}^{\omega}\left(t / \varepsilon^{2}\right)$ and $\bar{B}^{\varepsilon}(t)=\varepsilon \bar{B}\left(t / \varepsilon^{2}\right)$.

Let us recall some of the ideas of [13] and [4].

The process of the environment seen from the particle: $\left.\left(\omega(t)=X_{0}^{\omega}(t) . \omega\right) ; t \geq 0\right)$ is a Markov process under the annealed law, with values in $\Omega$. It is not difficult to check that the measure $\mathbb{Q}$ is invariant, ergodic and reversible for this process (Recall that $V=0$ for now!).

Given the state of the environment at times 0 and $t$, say $\omega(0)$ and $\omega(t)$, one retrieves the position of the particle itself by solving the equation $z . \omega(0)=\omega(t)$. Note that Assumptions 1 (either $V$ or $a$ is not constant) and 4 (independence property) imply that there cannot be more than one solution. It also follows from the equality $\left(X_{0}^{\omega}(t)-X_{0}^{\omega}(s)\right) . \omega(s)=\omega(t)$ that $X_{0}^{\omega}(t)$ is an antisymmetric additive functional of the process $\omega(\cdot)$. (Antisymmetric means that reversing time amounts to changing the sign of $X_{0}^{\omega}$.)

The process $\bar{B}$ is also an additive functional of $\omega(\cdot)$ since it can be written as the difference

$$
\begin{aligned}
\bar{B}(t)-\bar{B}(s) & =e_{1} \cdot\left(X_{0}^{\omega}(t)-X_{0}^{\omega}(s)\right)-\int_{s}^{t} e_{1} \cdot b^{\omega}\left(X_{0}^{\omega}(u)\right) d u \\
& =e_{1} \cdot\left(X_{0}^{\omega}(t)-X_{0}^{\omega}(s)\right)-\int_{s}^{t} e_{1} \cdot b(\omega(u)) d u
\end{aligned}
$$

see (2.4).

Sufficient conditions for invariance principles for additive functionals of reversible Markov processes that can be applied to $X_{0}^{\omega}$ or $\bar{B}$ are given in [13] and [4]. They yield a joint invariance principle for $\left(X_{0}^{\omega, \varepsilon}, \bar{B}^{\varepsilon}\right)$.

In order to compute the covariance matrix note that $X_{0}^{\omega}(t)$ is antisymmetric whereas $\int_{0}^{t} e_{1} \cdot b(\omega(s)) d s$ is a symmetric functional of the environment. Thus they are orthogonal under the annealed measure. Therefore

$$
\int E\left[\bar{B}(t) X_{0}^{\omega}(t)\right] d \mathbb{Q}=\int E\left[\left(e_{1} \cdot X_{0}^{\omega}(t)\right) X_{0}^{\omega}(t)\right] d \mathbb{Q},
$$

and thus the asymptotic covariance of $X_{0}^{\omega, \varepsilon}(1)$ and $\bar{B}^{\varepsilon}(1)$ coincides with the asymptotic covariance of $X_{0}^{\omega, \varepsilon}(1)$ and $e_{1} \cdot X_{0}^{\omega, \varepsilon}(1)$ and equals $\Sigma e_{1}$. 
Applying first the Girsanov formula and then the invariance principle, we get that, for any $\alpha>0$, as $\lambda \rightarrow 0$ and $t \rightarrow \infty$ with $\lambda^{2} t=\alpha$, we have

$$
\begin{aligned}
& \int E\left[F\left(\frac{\lambda}{\alpha} X_{0}^{\lambda, \omega}\left(\frac{\alpha}{\lambda^{2}} s\right) ; 0 \leq s \leq 1\right)\right] d \mathbb{Q} \\
= & \int E\left[F\left(\frac{\lambda}{\alpha} X_{0}^{\omega}\left(\frac{\alpha}{\lambda^{2}} s\right) ; 0 \leq s \leq 1\right) e^{\lambda \bar{B}\left(\frac{\alpha}{\lambda^{2}}\right)-\frac{\lambda^{2}}{2}\langle\bar{B}\rangle\left(\frac{\alpha}{\lambda^{2}}\right)}\right] d \mathbb{Q} \\
\rightarrow & E\left[F\left(\frac{1}{\sqrt{\alpha}} N(s) ; 0 \leq s \leq 1\right) e^{\sqrt{\alpha} Z(1)-\frac{\alpha}{2} E\left(Z(1)^{2}\right)}\right],
\end{aligned}
$$

where $F$ is a bounded continuous functional on $C\left([0,1], \mathbb{R}^{d}\right)$ and $(N, Z)$ is a centered Brownian motion of dimension $d+1$ with $N$ having $\Sigma$ as covariance matrix, and $E[Z(t) N(t)]=\Sigma e_{1} t$. We refer to Lemma 3.3 for the full justification of the passing to the limit in (3.6). Using now formula (3.3), we have:

$$
E\left[F\left(\frac{1}{\sqrt{\alpha}} N(s) ; 0 \leq s \leq 1\right) e^{\sqrt{\alpha} Z(1)-\frac{\alpha}{2} E\left(Z(1)^{2}\right)}\right]=E\left[F\left(\frac{1}{\sqrt{\alpha}} N(s)+\Sigma e_{1} s ; 0 \leq s \leq 1\right)\right] .
$$

We thus conclude that

$$
\begin{aligned}
& \mathbb{E}_{0}^{\lambda}\left[F\left(\frac{\lambda}{\alpha} X\left(\frac{\alpha}{\lambda^{2}} s\right) ; 0 \leq s \leq 1\right)\right]=\int E\left[F\left(\frac{\lambda}{\alpha} X_{0}^{\lambda, \omega}\left(\frac{\alpha}{\lambda^{2}} s\right) ; 0 \leq s \leq 1\right)\right] d \mathbb{Q} \\
& \rightarrow E\left[F\left(\frac{1}{\sqrt{\alpha}} N(s)+\Sigma e_{1} s ; 0 \leq s \leq 1\right)\right],
\end{aligned}
$$

i.e., when $\lambda^{2} t=\alpha$, the law of $\left((\lambda t)^{-1} X(t s) ; 0 \leq s \leq 1\right)$ under $\mathbb{P}_{0}^{\lambda}$ converges to the law of $\left(\frac{1}{\sqrt{\alpha}} N(s)+\right.$ $\left.\Sigma e_{1} s ; 0 \leq s \leq 1\right)$.

To finish the proof of the Proposition, we need a priori bounds on the moments of $|X(t)|$ under $\mathbb{P}_{0}^{\lambda}$. We shall prove in Lemma 4.5 that

$$
\limsup _{\lambda \rightarrow 0 ; t \rightarrow+\infty ; \lambda^{2} t=\alpha} E_{0}^{\lambda, \omega}\left[\frac{\max _{s \leq t}|X(s)|^{p}}{\lambda p^{p}}\right]<\infty,
$$

uniformly in $\omega$ and for all $p \geq 1$ and all $\alpha \geq 1$. Therefore

$$
\limsup _{\lambda \rightarrow 0 ; t \rightarrow+\infty ; \lambda^{2} t=\alpha} \mathbb{E}_{0}^{\lambda}\left[\frac{\max _{s \leq t}|X(s)|^{p}}{\lambda^{p} t^{p}}\right]<\infty,
$$

for all $p \geq 1$ and all $\alpha \geq 1$ and we observe that (3.7) together with the convergence of the law of $\left((\lambda t)^{-1} X(t s) ; 0 \leq s \leq 1\right)$ under $\mathbb{P}_{0}^{\lambda}$ to the law of $\left(\frac{1}{\sqrt{\alpha}} N(s)+\Sigma e_{1} s ; 0 \leq s \leq 1\right)$ implies (3.1) and (3.2). Indeed we have

$$
\frac{\max _{s \leq t}|X(s)|^{2}}{\lambda^{2} t^{2}}=\max _{s \leq 1}\left|\frac{\lambda}{\alpha} X\left(\frac{\alpha}{\lambda^{2}} s\right)\right|^{2}
$$

and therefore, with the notation above,

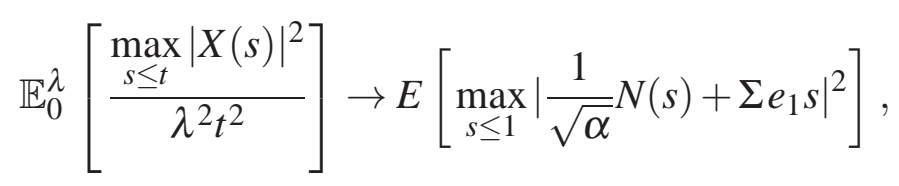


where we used the function $F(w(s) ; 0 \leq s \leq 1)=\max _{s \leq 1}|w(s)|^{2}$ and inequality (3.7) to justify the passing to the limit. Finally it is easy to check that

$$
\sup _{\alpha \geq 1} E\left[\max _{s \leq 1}\left|\frac{1}{\sqrt{\alpha}} N(s)+\Sigma e_{1} s\right|^{2}\right]<\infty .
$$

This last line ends the justification of (3.2). Equation (3.1) is proved the same way using now the function $F(w(s) ; 0 \leq s \leq 1)=w(1)$.

The proof of Proposition 3.1 is now complete in the case $V=0$.

Proof of Proposition 3.1 in the case $V \neq 0$

We do not assume anymore that $V=0$.

Define $Y^{\omega}$ and $Y^{\lambda, \omega}$ to be the solutions of the stochastic differential equations

$$
d Y^{\omega}(t)=e^{-2 V^{\omega}\left(Y^{\omega}(t)\right)} b^{\omega}\left(Y^{\omega}(t)\right) d t+e^{-V^{\omega}\left(Y^{\omega}(t)\right)} \sigma^{\omega}\left(Y^{\omega}(t)\right) d W_{t} ; Y^{\omega}(0)=0,
$$

and

$$
\begin{aligned}
d Y^{\lambda, \omega}(t)=\quad & e^{-2 V^{\omega}\left(Y^{\lambda, \omega}(t)\right)} b^{\omega}\left(Y^{\lambda, \omega}(t)\right) d t+e^{-2 V^{\omega}\left(Y^{\lambda, \omega}(t)\right)} a^{\omega}\left(Y^{\lambda, \omega}(t)\right) \hat{\lambda} d t \\
& +e^{-V^{\omega}\left(Y^{\lambda, \omega}(t)\right)} \sigma^{\omega}\left(Y^{\lambda, \omega}(t)\right) d W_{t} ; Y^{\lambda, \omega}(0)=0,
\end{aligned}
$$

so that the generators of $Y^{\omega}$ and $Y^{\lambda, \omega}$ are the operators

$$
\mathscr{M}^{\omega} f(x)=\frac{1}{2} \operatorname{div}\left(e^{-2 V^{\omega}} a^{\omega} \nabla f\right)(x),
$$

and

$$
\mathscr{M}^{\lambda, \omega} f(x)=\frac{1}{2} e^{\hat{\lambda} \cdot x} \operatorname{div}\left(e^{-2 V^{\lambda, \omega}} a^{\omega} \nabla f\right)(x),
$$

where $V^{\lambda, \omega}(x)=V^{\omega}(x)-\hat{\lambda} \cdot x$.

Note that these operators are of the same form as $\mathscr{L}^{\omega}$ and $\mathscr{L}^{\lambda, \omega}$ with $V^{\omega}$ being replaced by 0 and $a^{\omega}$ being replaced by $\exp \left(-2 V^{\omega}\right) a^{\omega}$. Thus we may apply the results obtained in the special case of a vanishing potential to the processes $Y^{\omega}$ and $Y^{\lambda, \omega}$, in particular $Y^{\omega}$ satisfies the invariance principle with some asymptotic diffusivity $\Sigma^{Y}$ and $Y^{\lambda, \omega}$ satisfies:

$$
\lim _{\lambda \rightarrow 0 ; t \rightarrow+\infty ; \lambda^{2} t=\alpha} \int_{\Omega} E\left[\frac{Y^{\lambda, \omega}(t)}{\lambda t}\right] d \mathbb{Q}=\Sigma^{Y} e_{1},
$$

and

$$
\sup _{\alpha \geq 1} \limsup _{\alpha \rightarrow 0 ; t \rightarrow+\infty ; \lambda^{2} t=\alpha} \int_{\Omega} E\left[\frac{\max _{s \leq t}\left|Y^{\lambda, \omega}(s)\right|^{2}}{\lambda^{2} t^{2}}\right] d \mathbb{Q}<\infty
$$

Fix $\omega$ and set

$$
A^{\omega}(t)=\int_{0}^{t} e^{-2 V^{\omega}\left(Y^{\omega}(s)\right)} d s=\int_{0}^{t} e^{-2 V\left(Y^{\omega}(s) \cdot \omega\right)} d s .
$$


Then $X_{0}^{\omega}$ has the same law as the time changed process $Y^{\omega}\left(\left(A^{\omega}\right)^{-1}\right)$. Similarly, if we let

$$
A^{\lambda, \omega}(t)=\int_{0}^{t} e^{-2 V^{\omega}\left(Y^{\lambda, \omega}(s)\right)} d s
$$

then $X_{0}^{\lambda, \omega}$ has the same law as the time changed process $Y^{\lambda, \omega}\left(\left(A^{\lambda, \omega}\right)^{-1}\right)$.

¿From Assumption 3, we know that $V$ is bounded and therefore

$$
A^{\lambda, \omega}(t) \geq \underline{c} t,
$$

for some constant $\underline{c} \leq 1$. Observe that (3.2) immediately follows from (3.12) and (3.11).

Proof of (3.1)

Let

$$
\gamma=\int d \mathbb{Q}(\omega) e^{-2 V(\omega)}
$$

The ergodic theorem for the process $Y^{\omega}$. $\omega$ implies that $A^{\omega}(t) / t$ almost surely converges to $\gamma$. We need a similar statement for $A^{\lambda, \omega}$ :

Lemma 3.4 For fixed $\alpha>0$ and any positive $\eta$, we have $\mathbb{Q}-$ a.s.

$$
P\left[\left|\frac{A^{\lambda, \omega}(t)}{t}-\gamma\right| \geq \eta\right] \rightarrow 0
$$

for $\lambda \rightarrow 0, t \rightarrow+\infty, \lambda^{2} t=\alpha$.

\section{Proof of Lemma 3.4}

Use the Girsanov formula (3.4) to see that

$$
P\left[\left|\frac{A^{\lambda, \omega}(t)}{t}-\gamma\right| \geq \eta\right]=E\left[\mathbf{1}\left(\left|\frac{A^{\omega}(t)}{t}-\gamma\right| \geq \eta\right) e^{\lambda \bar{B}(t)-\frac{\lambda^{2}}{2}\langle\bar{B}\rangle(t)}\right] .
$$

The convergence in (3.13) then follows from Hölder's inequality, the bound in Lemma 3.3 and the fact that $P\left[\left|\frac{A^{\omega}(t)}{t}-\gamma\right| \geq \eta\right] \rightarrow 0$.

Back to the proof of (3.1), we start with the equality

$$
\mathbb{E}_{0}^{\lambda}\left[\frac{X(t)}{\lambda t}\right]=\int_{\Omega} E\left[\frac{Y^{\lambda, \omega}\left(\left(A^{\lambda, \omega}\right)^{-1}(t)\right)}{\lambda t}\right] d \mathbb{Q}
$$

We have

$$
\begin{aligned}
& \left|\int_{\Omega} E\left[\frac{Y^{\lambda, \omega}\left(\left(A^{\lambda, \omega}\right)^{-1}(t)\right)}{\lambda t}\right] d \mathbb{Q}-\int_{\Omega} E\left[\frac{Y^{\lambda, \omega}(t / \gamma)}{\lambda t}\right] d \mathbb{Q}\right| \\
\leq & \int_{\Omega} E\left[\frac{\max _{\Omega-t / \gamma \mid \leq \eta t}\left|Y^{\lambda, \omega}(s)-Y^{\lambda, \omega}(t / \gamma)\right|}{\lambda t}\right] d \mathbb{Q} \\
+ & \int_{\Omega} E\left[\frac{\max _{s \leq t / \underline{c}}\left|Y^{\lambda, \omega}(s)\right|}{\lambda t} \mathbf{1}\left(\left|\left(A^{\lambda, \omega}\right)^{-1}(t)-t / \gamma\right| \geq \eta t\right)\right] d \mathbb{Q} \\
= & I+I I .
\end{aligned}
$$


( $\underline{c}$ is the same constant as in (3.12). Note that $\underline{c} \leq \gamma$.)

By the Markov property, we have

$$
I \leq 2 \int_{\Omega} E\left[\frac{\max _{s \leq 2 \eta t}\left|Y^{\lambda, \omega}(s)\right|}{\lambda t}\right] d \mathbb{Q} .
$$

Now (3.11) implies that, if we let $\lambda$ tend to 0 and then $\eta$ tend to 0 , then the contribution of I vanishes. Hölder's inequality, (3.11) again and Lemma 3.4 imply that, for any $\eta>0$, then II also converges to 0 as $t$ tends to $+\infty$. We conclude that

$$
\lim _{\lambda \rightarrow 0 ; t \rightarrow+\infty ; \lambda^{2} t=\alpha} \int_{\Omega}\left(E\left[\frac{Y^{\lambda, \omega}\left(\left(A^{\lambda, \omega}\right)^{-1}(t)\right)}{\lambda t}\right]-E\left[\frac{Y^{\lambda, \omega}(t / \gamma)}{\lambda t}\right]\right) d \mathbb{Q}=0
$$

and, using (3.10),

$$
\lim _{\lambda \rightarrow 0 ; t \rightarrow+\infty ; \lambda^{2} t=\alpha} \mathbb{E}_{0}^{\lambda}\left[\frac{X(t)}{\lambda t}\right]=\frac{1}{\gamma} \Sigma^{Y} e_{1} .
$$

The last piece of information missing is the equality $\Sigma^{Y}=\gamma \Sigma$. It comes as follows: since $\frac{1}{\sqrt{t}} Y^{\omega}(t)$ converges in law to a Gaussian random variable with covariance $\Sigma^{Y}$ and since $\frac{1}{t} A^{\omega}(t)$ almost surely converges to $\gamma$ then $\frac{1}{\sqrt{t}} X_{0}^{\omega}(t)=\frac{1}{\sqrt{t}} Y^{\omega}\left(\left(A^{\omega}\right)^{-1}(t)\right)$ converges in law to a Gaussian random variable with covariance $\Sigma=\frac{1}{\gamma} \Sigma^{Y}$.

\section{A priori estimates}

In this section, we prove some a priori estimates on exit times that quantify the fact that the process $X^{\lambda, \omega}$ is transient in the direction $e_{1}$.

For a given realization of the environment, the local drift of the process $X^{\omega}$ equals $b^{\omega}\left(X^{\omega}(t)\right)$. Its mean under the annealed law vanishes. The drift of $X^{\lambda, \omega}$ has an extra $a^{\omega}\left(X^{\lambda, \omega}(t)\right) \hat{\lambda}$ term. Since, by Assumption 3 (uniform ellipticity), we have $e_{1} \cdot a^{\omega}(x) e_{1} \geq \kappa\left|e_{1}\right|^{2} \neq 0$ for any $\omega$ and $x$, one would expect $X^{\lambda, \omega}$ to be transient in the direction $e_{1}$, and this turns out to be the case, but we also need more quantitative statements on the tendency of the diffusion to go in the direction $e_{1}$.

Roughly speaking, we may think of $e_{1} \cdot X^{\lambda, \omega}(t)$ as the sum of a centered term of order $\sqrt{t}$ and a drift term of order $\lambda t$. Thus the shortest scale on which we may hope the drift term to dominate is $\lambda^{2} t \geq 1$ or, in terms of space scale, $\lambda L \geq 1$. Up to the value of the constants $\kappa_{1}, \kappa_{2}$, $c$ and $C$, our estimates in Lemma 4.1 and Lemma 4.2 are therefore optimal.

In the following Lemmata, inequalities (4.1) and (4.2) hold true for any environment $\omega$ satisfying Assumptions 2 and 3. Assumptions 1 and 4 are not relevant in this section.

We use $T_{L}=\inf \left\{t: e_{1} \cdot X(t)=L\right\}$ to denote the hitting time of the hyperplane $\left\{x: e_{1} \cdot x=L\right\}$, $L \in \mathbb{R}$.

Lemma 4.1 There exists constants $c>0$ and $\kappa_{1}>0$ that depend on the dimension, the ellipticity constant $\kappa$ and the $L^{\infty}$ bound on $V$ such that for all $L, \lambda \leq 1$ and for any environment $\omega$,

$$
P_{0}^{\lambda, \omega}\left[T_{-L}<\infty\right] \leq c e^{-\kappa_{1} \lambda L}
$$


Lemma 4.2 There exists constants $C$ and $\kappa_{2}>0$ that depend on the dimension, the ellipticity constant $\kappa$ and the $L^{\infty}$ bound on $V$ such that for all $L, \lambda \leq 1, t \geq 0$ and for any environment $\omega$,

$$
P_{0}^{\lambda, \omega}\left[T_{L} \geq t\right] \leq C e^{-\kappa_{2} \lambda^{2} t+\lambda L}
$$

Proof of Lemma 4.1.

Let $u(x)=u_{\lambda, \omega, L}(x):=P_{x}^{\lambda, \omega}\left[T_{L}<T_{-L}\right]$. Then, $u$ solves the elliptic boundary value problem

$$
\mathscr{L}^{\lambda, \omega} u(x)=0, \quad-L \leq e_{1} \cdot x \leq L
$$

where the generator $\mathscr{L}^{\lambda, \omega}$ was defined in (2.8), with boundary values

$$
u(x)=0 \text { if } e_{1} \cdot x=-L \text { and } u(x)=1 \text { if } e_{1} \cdot x=L .
$$

We first need the following Lemma, whose proof is deferred.

Lemma 4.3 Assume $\lambda=1$ and $u$ is the solution of the boundary value problem above. Then, under Assumption 3, there exists $L_{0}>0$ depending only on $\kappa$ and on the dimension, such that for all $L \geq L_{0}$ and all $\omega$,

$$
u(x) \geq \frac{2}{3} \quad \text { for all } x \text { with } e_{1} \cdot x=0
$$

Let $\widetilde{X}(t)=\lambda X\left(\frac{t}{\lambda^{2}}\right), t \geq 0$. Then, $(\widetilde{X}(t))_{t \geq 0}$ is a Markov process with generator

$$
\widetilde{\mathscr{L}}^{\lambda, \omega} f(x)=\frac{1}{2} e^{2 V^{\omega}(x / \lambda)} \operatorname{div}\left(e^{-2 V^{\omega}(x / \lambda)} a^{\omega}(x / \lambda) \nabla f\right)(x)+a^{\omega}(x / \lambda) e_{1} \cdot \nabla f(x)
$$

(The advantage of $(\widetilde{X}(t))_{t \geq 0}$ is that we scaled away the drift and we will be able to apply Lemma 4.3). Let us consider exit times for $(\widetilde{X}(t))_{t \geq 0}$.

$$
\widetilde{T}_{L}=\inf \left\{t: e_{1} \cdot \widetilde{X}(t)=L\right\}, \quad \widetilde{T}_{-L}=\inf \left\{t: e_{1} \cdot \widetilde{X}(t)=-L\right\},
$$

and

$$
\widetilde{T}_{ \pm L}=\inf \left\{t:\left|e_{1} \cdot \widetilde{X}(t)\right|=L\right\}
$$

Then,

$$
P_{0}^{\lambda, \omega}\left[T_{-L}<\infty\right]=P_{0}^{\lambda, \omega}\left[\widetilde{T}_{-\lambda L}<\infty\right]
$$

Hence, it suffices to show that for all $\lambda, L, \omega$, we have $P_{0}^{\lambda, \omega}\left[\widetilde{T}_{-\lambda L}<\infty\right] \leq c e^{-\kappa_{1} \lambda L}$ which in turn is equivalent to

$$
P_{0}^{\lambda, \omega}\left[\widetilde{T}_{-L}<\infty\right] \leq c e^{-\kappa_{1} L},
$$

for all $\lambda, L, \omega$. Let $L_{0}$ be as in Lemma 4.3. We consider the embedded random walk defined as follows. Let $t_{1}:=\widetilde{T}_{ \pm L_{0}}, t_{i+1}=\inf \left\{t \geq t_{i}:\left|e_{1} \cdot\left(\widetilde{X}(t)-X\left(t_{i}\right)\right)\right|=L_{0}\right\}$ and $S_{i}=\widetilde{X}_{t_{i}}, i=1,2, \ldots$, (and $\left.S_{0}=0\right)$. Due to Lemma 4.3 and the strong Markov property of $(\widetilde{X}(t))_{t \geq 0}$, we have

$$
P_{0}^{\lambda, \omega}\left[S_{i+1}=S_{i}+L_{0}\right] \geq \frac{2}{3}
$$


Hence we can couple $\left(S_{i}\right)_{i=0,1,2, \ldots}$ with a standard random walk with drift $\left(\bar{S}_{i}\right)_{i=0,1,2, \ldots}$ on $L_{0} \mathbb{Z}$ with iid increments, satisfying $P\left[\bar{S}_{i+1}=\bar{S}_{i}+L_{0}\right]=\frac{2}{3}=1-P\left[\bar{S}_{i+1}=\bar{S}_{i}-L_{0}\right]$ and the coupling is such that $S_{i} \geq \bar{S}_{i}$ for all $i$. Explicit calculation yields

$$
P\left[\bar{S}_{i}>-m L_{0}, \forall i\right]=1-\frac{1}{2^{m}}
$$

and we obtain

$$
P_{0}^{\lambda, \omega}\left[S_{i}>-m L_{0}, \forall i\right] \geq 1-\frac{1}{2^{m}}
$$

which implies (4.6).

Proof of Lemma 4.2 .

Inequality (4.2) is equivalent to the following statement in terms of $(\widetilde{X}(t))_{t \geq 0}$ : There exist constants $C$ and $\kappa_{2}>0$ that depend on the dimension, the ellipticity constant $\kappa$ and the $L^{\infty}$ bound on $V$ such that for all $L$ and $t$ and for any environment $\omega$,

$$
P_{0}^{\lambda, \omega}\left[\widetilde{T}_{L} \geq t\right] \leq C e^{-\kappa_{2} t+L}
$$

Indeed, note that $\widetilde{T}_{\lambda L}=\lambda^{2} T_{L}$.

Take $L_{0}$ as in Lemma 4.3. Then Aronson's estimate (see [1]) yields that for all $x \in \Pi_{L_{0}}:=\{x$ : $\left.-L_{0} \leq e_{1} \cdot x \leq L_{0}\right\}$

$$
P_{x}^{\lambda, \omega}\left[\left|e_{1} \cdot \widetilde{X}(1)\right|>L_{0}\right] \geq \gamma>0,
$$

where $\gamma$ only depends on $L_{0}, \kappa$ and the dimension. Indeed, according to [1], the fundamental solution $\mathscr{P}(x, y, t)$ of the parabolic operator

$$
\partial_{t}-\operatorname{div}(a(x) \nabla)-a(x) e_{1} \cdot \nabla
$$

(or, equivalently, transition probability density of the corresponding diffusion) satisfies the lower bound

$$
\mathscr{P}(x, y, 1) \geq k \exp \left(-K|x-y|^{2}\right)
$$

with constants $k>0$ and $K>0$ which only depend on $\kappa$ and $d$. The estimate (4.8) is an immediate consequence of this lower bound. Due to the Markov property of $(\widetilde{X}(t))_{t \geq 0}$, estimate (4.8) implies that for some constant $g_{0}$ (which depends only on $L_{0}, \kappa$ and the dimension),

$$
P_{x}^{\lambda, \omega}\left[\widetilde{T}_{ \pm L_{0}} \geq g_{0}\right] \leq \frac{1}{15}
$$

for all $x \in \Pi_{L_{0}}$. Define the stopping time $\widetilde{t}$ as follows:

$$
\widetilde{t}:=\inf \left\{t \geq 0:\left|e_{1} \cdot(\widetilde{X}(t)-\widetilde{X}(0))\right|=L_{0}\right\} \wedge g_{0} .
$$

Combining (4.9) with Lemma 4.3, we obtain

$$
P_{x}^{\lambda, \omega}\left[e_{1} \cdot \tilde{X}(\widetilde{t})=e_{1} \cdot x+L_{0}\right] \geq \frac{3}{5}
$$

(with probability at least $\frac{14}{15}$, the layer $\left\{y:\left|e_{1} \cdot y-e_{1} \cdot x\right|<L_{0}\right\}$ has been left by time $g_{0}$, and with probability at least $\frac{2}{3}$, the exit happens at $\left\{y: e_{1} \cdot y=e_{1} \cdot x+L_{0}\right\}$ ). We consider the embedded 
random walk defined as follows. Let $t_{1}:=\widetilde{t}, t_{i+1}=\widetilde{t} \circ \theta_{t_{i}}+t_{i}, S_{i}=e_{1} \cdot \widetilde{X}\left(t_{i}\right), i=1,2, \ldots$ and $S_{0}=0$. (Here $\theta$ denotes the shift operator on path space). Hence we can couple $\left(S_{i}\right)_{i=0,1,2, \ldots}$ with a standard random walk with drift $\left(\bar{S}_{i}\right)_{i=0,1,2, \ldots}$ on $L_{0} \mathbb{Z}$ with i.i.d. increments, satisfying $P\left[\bar{S}_{i+1}=\bar{S}_{i}+L_{0}\right]=\frac{3}{5}=$ $1-P\left[\bar{S}_{i+1}=\bar{S}_{i}-L_{0}\right]$ and the coupling is such that $S_{i} \geq \bar{S}_{i}$ for all $i$. It is straightforward to check that there are constants $\kappa_{3}>0$ and $c>0$ such that for all $n \in \mathbb{N}$ and $L \in \mathbb{R}$,

$$
P\left[\bar{S}_{n} \leq L L_{0}\right] \leq c e^{-\kappa_{3} n+L} .
$$

We conclude, by comparison, that we have for all $L \in \mathbb{R}$ and $n \in \mathbb{N}$

$$
P_{0}^{\lambda, \omega}\left[\widetilde{T}_{L L_{0}} \geq n g_{0}\right] \leq P_{0}^{\lambda, \omega}\left[S_{n} \leq L L_{0}\right] \leq P\left[\bar{S}_{n} \leq L L_{0}\right] \leq c e^{-\kappa_{3} n+L},
$$

and this implies (4.7).

Proof of Lemma 4.3.

Without loss of generality we can assume that $V=0$. Indeed, multiplying (4.3) by $\exp \left(-2 V^{\omega}(x)\right)$ and denoting $a(x)=\exp \left(-2 V^{\omega}(x)\right) a^{\omega}(x)$ one can rewrite equations (4.3)-(4.4) in the form

$$
\begin{gathered}
\operatorname{div}(a(x) \nabla u)+a(x) e_{1} \cdot \nabla u=0 \quad \text { in } \Pi, \\
u(-L, z)=0, \quad u(L, z)=1 .
\end{gathered}
$$

with $x=\left(x_{1}, z\right)$ and $\Pi=\Pi_{L}=\left\{x \in \mathbb{R}^{d}:-L \leq x_{1} \leq L\right\}$. Under Assumption 3 the matrix $a(x)$ is symmetric and satisfies the following elliptic estimates

$$
\kappa|y|^{2} \leq a(x) y \cdot y \leq \kappa^{-1}|y|^{2}, \quad \forall x, y \in \mathbb{R}^{d} .
$$

Therefore, it suffices to prove the following statement.

Lemma 4.4 There is $L_{0}=L_{0}(\kappa, d)$ such that for all $L \geq L_{0}$, we have

$$
u(0, z) \geq 2 / 3, \quad z \in \mathbb{R}^{d-1} .
$$

\section{Proof of Lemma 4.4}

Suppose that $u(0, z)<2 / 3$ for some $z \in \mathbb{R}^{d-1}$ and some $L$. Without loss of generality we assume that $z=0$. The function $1-u(x)$ is a non-negative solution of the equation

$$
\operatorname{div}(a(x) \nabla(1-u))+a(x) e_{1} \cdot \nabla(1-u)=0,
$$

therefore, by the Harnack inequality (see, for instance, [10]) there is a constant $C=C(\kappa, d)$ such that

$$
1-u(0, z) \geq C(\kappa, d)(1-u(0)) \geq \frac{1}{3} C(\kappa, d), \quad z \in[-1,1]^{d-1} .
$$

For all $z \in[-1,1]^{d-1}$ this implies the estimate

$$
\frac{1}{9} C^{2}(\kappa, d) \leq\left(\int_{0}^{L} \frac{\partial\left(1-u\left(x_{1}, z\right)\right)}{\partial x_{1}} d x_{1}\right)^{2} \leq L \int_{0}^{L}\left(\frac{\partial u\left(x_{1}, z\right)}{\partial x_{1}}\right)^{2} d x_{1}
$$




$$
\leq L \int_{0}^{N}\left|\nabla u\left(x_{1}, z\right)\right|^{2} d x_{1} \leq L \int_{-L}^{L} e^{x_{1}}\left|\nabla u\left(x_{1}, z\right)\right|^{2} d x_{1}
$$

where we used Jensen's inequality for the second inequality. Integrating over $[-1,1]^{d-1}$, we obtain

$$
\int_{-L}^{L} d x_{1} \int_{[-1,1]^{d-1}} e^{x_{1}}\left|\nabla u\left(x_{1}, z\right)\right|^{2} d z \geq \frac{1}{L} C_{1}(\kappa, d),
$$

with a constant $C_{1}(\kappa, d)>0$ which only depends on $\kappa$ and $d$. For arbitrary sets $G \subseteq \Pi$ and functions $v(x)$, we write $\mathscr{E}(v, G)$ for the energy of $v$ on $G$ :

$$
\mathscr{E}(v, G)=\int_{G} e^{x_{1}} a(x) \nabla v(x) \cdot \nabla v(x) d x
$$

Hence, (4.13) can be rewritten, setting $G_{0}=[-L, L] \times[-1,1]^{d-1}$, as follows:

$$
\mathscr{E}\left(u, G_{0}\right) \geq \frac{1}{L} C_{1}(\kappa, d)
$$

(The value of $C_{1}(\kappa, d)$ changes from equation (4.13) to equation (4.14).) We will give an upper bound for $\mathscr{E}\left(u, G_{0}\right)$ which contradicts (4.14) when $L$ is too large. We introduce the following subsets of $\Pi$.

$$
\begin{gathered}
B_{0}=\left[-e^{L / d}, e^{L / d}\right]^{d-1}, \quad \Pi_{0}=[-L, L] \times B_{0}, \\
B_{j}=2^{-j} B_{0}=2^{-j}\left[-e^{L / d}, e^{L / d}\right]^{d-1}, \quad \Pi_{j}=[-L, L] \times B_{j},
\end{gathered}
$$

Also, denote

$$
\bar{u}(x)=\bar{u}\left(x_{1}\right)= \begin{cases}1, & \text { if } x_{1} \geq-L+1, \\ x+L, & \text { if }-L \leq x_{1} \leq-L+1\end{cases}
$$

and, writing again $x=\left(x_{1}, z\right)$,

$$
v_{j}(x)= \begin{cases}\left(1-d_{j}(z)\right) u(x)+d_{j}(z) \bar{u}(x), & \text { if } d_{j}(z) \leq 1 \\ \bar{u}(x), & \text { otherwise }\end{cases}
$$

where

$$
d_{j}(z)=2^{j+1} e^{-L / d} \operatorname{dist}_{\mathbb{R}^{d-1}}\left(z, \partial B_{j}\right), \quad j=0,1, \ldots, 2 d .
$$

Note that $v_{j}=\bar{u}$ in the domain $\Pi_{j+1}$. Since $v_{0}=u$ on $\partial \Pi_{0}$, we know that

$$
\mathscr{E}\left(u, \Pi_{0}\right) \leq \mathscr{E}\left(v_{0}, \Pi_{0}\right)
$$

Indeed, the functional $\left\{\mathscr{E}\left(v, \Pi_{0}\right): v \in H^{1}\left(\Pi_{0}\right),\left.v\right|_{\partial \Pi_{0}}=\left.u\right|_{\partial \Pi_{0}}\right\}$ attains its minimum at the unique solution of the equation

$$
\operatorname{div}(a(x) \nabla v)+a(x) e_{1} \nabla v=0 \quad \text { in } \Pi_{0},\left.\quad v\right|_{\partial \Pi_{0}}=\left.u\right|_{\partial \Pi_{0}} .
$$

Clearly, the function $u$ solves this equation, and the required inequality follows. 
For $x \in \Pi_{0} \backslash \Pi_{1}$ we have

$$
\begin{gathered}
e^{x_{1}} a(x) \nabla v_{0}(x) \cdot \nabla v_{0}(x) \\
=e^{x_{1}} a(x)\left\{\left(1-d_{0}(x)\right) \nabla u(x)+d_{0}(x) \nabla \bar{u}(x)\right\} \cdot\left\{\left(1-d_{0}(x)\right) \nabla u(x)+d_{0}(x) \nabla \bar{u}(x)\right\} \\
+2 e^{x_{1}} a(x)\left(\nabla d_{0}(x)\right)(\bar{u}(x)-u(x)) \cdot\left\{\left(1-d_{0}(x)\right) \nabla u(x)+d_{0}(x) \nabla \bar{u}(x)\right\} \\
+e^{x_{1}} a(x)\left(\nabla d_{0}(x)\right)(\bar{u}(x)-u(x)) \cdot\left(\nabla d_{0}(x)\right)(\bar{u}(x)-u(x))
\end{gathered}
$$

Using the convexity of the scalar product, this is

$$
\begin{gathered}
\leq\left(1-d_{0}(x)\right) e^{x_{1}} a(x) \nabla u(x) \cdot \nabla u(x)+d_{0}(x) e^{x_{1}} a(x) \nabla \bar{u}(x) \cdot \nabla \bar{u}(x) \\
\quad+2 e^{x_{1}} \kappa^{-1}\left|\nabla d_{0}(x)\right||\bar{u}(x)-u(x)|(|\nabla u(x)|+|\nabla \bar{u}(x)|) \\
+e^{x_{1}} a(x)\left(\nabla d_{0}(x)\right)(\bar{u}(x)-u(x)) \cdot\left(\nabla d_{0}(x)\right)(\bar{u}(x)-u(x)) .
\end{gathered}
$$

For $x \in \Pi_{1}$, we have

$$
e^{x_{1}} a(x) \nabla v_{0}(x) \cdot \nabla v_{0}(x)=e^{x_{1}} a(x) \nabla \bar{u}(x) \cdot \nabla \bar{u}(x) .
$$

After integrating the former inequality over $\Pi_{0} \backslash \Pi_{1}$ and the latter over $\Pi_{1}$, and summing up, we get

$$
\begin{gathered}
\mathscr{E}\left(u, \Pi_{0}\right) \leq \mathscr{E}\left(v_{0}, \Pi_{0}\right) \leq \mathscr{E}\left(u, \Pi_{0} \backslash \Pi_{1}\right)+\mathscr{E}\left(\bar{u}, \Pi_{0}\right) \\
+4 \kappa^{-1} e^{-L / d} \int_{\Pi_{0} \backslash \Pi_{1}} e^{x_{1}}|\bar{u}(x)-u(x)|(|\nabla u(x)|+|\nabla \bar{u}(x)|) d x \\
+4 \kappa^{-1} e^{-2 L / d} \int_{\Pi_{0} \backslash \Pi_{1}} e^{x_{1}}(u(x)-u(x))^{2} d x .
\end{gathered}
$$

After simple rearrangements this yields, using Hölder's inequality,

$$
\begin{gathered}
\mathscr{E}\left(u, \Pi_{1}\right) \leq \mathscr{E}\left(\bar{u}, \Pi_{0}\right)+4 \kappa^{-1} e^{-2 L / d} \int_{\Pi_{0} \backslash \Pi_{1}} e^{x_{1}}(u(x)-u(x))^{2} d x \\
+4 \kappa^{-1} e^{-L / d}\left(\int_{\Pi_{0} \backslash \Pi_{1}} e^{x_{1}}(u(x)-u(x))^{2} d x\right)^{1 / 2}\left(\int_{\Pi_{0} \backslash \Pi_{1}} e^{x_{1}}\left(|\nabla \bar{u}(x)|^{2}+|\nabla u(x)|^{2}\right) d x\right)^{1 / 2}
\end{gathered}
$$

Our next aim is to estimate the integrals on the right-hand side of the last inequality in terms of the energies of $u$ and $\bar{u}$.

First, we estimate

$$
\int_{\Pi_{0} \backslash \Pi_{1}} e^{x_{1}}\left((u(x)-u(x))^{2} d x \leq \int_{\Pi_{0} \backslash \Pi_{1}} e^{x_{1}}(u(x)-1)^{2} d x+\int_{\Pi_{0} \backslash \Pi_{1}} e^{x_{1}}(1-u(x))^{2} d x .\right.
$$

Since $(u-1)=0$ for $x_{1}=L$, we have

$$
(u(x)-1)^{2}=\left(\int_{x_{1}}^{L} \frac{\partial u}{\partial x_{1}}(y, z) d y\right)^{2} \leq 2 L \int_{x_{1}}^{L}|\nabla u(y, z)|^{2} d y,
$$


where we used Jensen's inequality. Multiplying this bound by $e^{x_{1}}$ and integrating over the set $\Pi_{0} \backslash \Pi_{1}$, we obtain

$$
\begin{aligned}
\int_{\Pi_{0} \backslash \Pi_{1}} e^{x_{1}}(1-u(x))^{2} d x & \leq \int_{\Pi_{0} \backslash \Pi_{1}} e^{x_{1}} 2 L \int_{x_{1}}^{L}|\nabla u(y, z)|^{2} d y d x \\
& \leq \int_{\Pi_{0} \backslash \Pi_{1}} 2 L \int_{x_{1}}^{L} e^{y}|\nabla u(y, z)|^{2} d y d z \\
& \leq 4 L^{2} \int^{x^{x_{1}}|\nabla u(x)|^{2} d x}
\end{aligned}
$$

Similarly, taking into account that $\bar{u} \neq 1$ only for $x_{1} \leq-L+1$, we obtain

$$
\int_{\Pi_{0} \backslash \Pi_{1}} e^{x_{1}}(1-\bar{u}(x))^{2} d x \leq \int_{\Pi_{0} \backslash \Pi_{1} \cap\left\{x_{1} \leq-L+1\right\}} e^{x_{1}} d x \leq(e-1) 2^{d-1} e^{-L} e^{(d-1) L / d}=a_{d} e^{-\frac{L}{d}},
$$

where $a_{d}=(e-1) 2^{d-1}$. Combining the latter bound with (4.16) yields

$$
\begin{aligned}
\int_{\Pi_{0} \backslash \Pi_{1}} e^{x_{1}}\left((u(x)-u(x))^{2} d x\right. & \leq a_{d} e^{-\frac{L}{d}}+4 L^{2} \int_{\Pi_{0} \backslash \Pi_{1}} e^{x_{1}}|\nabla u(x)|^{2} d x \\
& \leq a_{d} e^{-\frac{L}{d}}+4 L^{2} \kappa^{-1} \int_{\Pi_{0} \backslash \Pi_{1}} e^{x_{1}} a(x) \nabla u(x) \cdot \nabla u(x) d x \\
& =a_{d} e^{-\frac{L}{d}}+4 L^{2} \kappa^{-1} E\left(u, \Pi_{0} \backslash \Pi_{1}\right)
\end{aligned}
$$

In the same way, using $|\nabla \bar{u}(x)|=\mathbf{1}_{\left\{x_{1} \leq-L+1\right\}}$, for the last integral on the right-hand side of (4.15) we have

$$
\int_{\Pi_{0} \backslash \Pi_{1}} e^{x_{1}}\left(|\nabla \bar{u}(x)|^{2}+|\nabla u(x)|^{2}\right) d x \leq a_{d} e^{-\frac{L}{d}}+\Lambda^{-1} E\left(u, \Pi_{0} \backslash \Pi_{1}\right)
$$

The following bound for $\mathscr{E}\left(\bar{u}, \Pi_{0}\right)$ is straightforward:

$$
\mathscr{E}\left(\bar{u}, \Pi_{0}\right) \leq \kappa^{-1} \int_{\Pi_{0}} e^{x_{1}}|\nabla \bar{u}(x)|^{2} d x \leq \kappa^{-1} a_{d} e^{-\frac{L}{d}} .
$$

¿From (4.15), (4.17), (4.18) and the last bound, we derive the inequality

$$
\begin{aligned}
\mathscr{E}\left(u, \Pi_{1}\right) \leq & \kappa^{-1} a_{d} e^{-\frac{L}{d}}+\kappa^{-1} e^{-2 L / d}\left(a_{d} e^{-\frac{L}{d}}+4 L^{2} \kappa^{-1} \mathscr{E}\left(u, \Pi_{0} \backslash \Pi_{1}\right)\right) \\
& +4 \kappa^{-1} e^{-L / d}\left(a_{d} e^{-\frac{L}{d}}+4 L^{2} \kappa^{-1} \mathscr{E}\left(u, \Pi_{0} \backslash \Pi_{1}\right)\right)^{1 / 2} \\
& \cdot\left(a_{d} e^{-\frac{L}{d}}+\kappa^{-1} \mathscr{E}\left(u, \Pi_{0} \backslash \Pi_{1}\right)\right)^{1 / 2} \\
\leq & C_{\kappa, d}\left(e^{-\frac{L}{d}}+L^{2} e^{-L / d} \mathscr{E}\left(u, \Pi_{0} \backslash \Pi_{1}\right)\right) \leq C_{\kappa, d}\left(e^{-\frac{L}{d}}+L^{2} e^{-L / d} \mathscr{E}\left(u, \Pi_{0}\right)\right)
\end{aligned}
$$

Let us now estimate the energy $\mathscr{E}\left(u, \Pi_{0}\right)$. To this end we denote $G(r, x)=x+[-r, r]^{d}$, and notice that, by the standard elliptic estimates (see [10]),

$$
\|\nabla u\|_{L^{2}(G(1, x) \cap \Pi)} \leq C_{1}(\kappa, d)\|u\|_{L^{2}(G(2, x) \cap \Pi)} \leq C_{2}(\kappa, d)
$$


with a constant $C_{2}(\kappa, d)$ which depends only on $\kappa$ and the dimension. This implies the bound

$$
\mathscr{E}\left(u, \Pi_{0}\right) \leq C_{4}(\kappa, d) e^{L} e^{(d-1) L / d}=C_{4}(\kappa, d) e^{(2 d-1) L / d} .
$$

Together with (4.19) this gives

$$
\mathscr{E}\left(u, \Pi_{1}\right) \leq C_{5}(\kappa, d) L^{2} e^{(2 d-2) L / d}
$$

In exactly the same way as (4.19), we obtain (for $L^{2}>C_{5}(\kappa, d)$ )

$$
\mathscr{E}\left(u, \Pi_{2}\right) \leq C_{5}(\kappa, d)\left(L^{2} e^{-L / d} E\left(u, \Pi_{1}\right)+e^{-\frac{L}{d}}\right) \leq C_{5}(\kappa, d) L^{4} e^{(2 d-3) L / d} .
$$

Iterating this procedure $2 d$ times we finally get

$$
\mathscr{E}\left(u, \Pi_{2 d}\right) \leq C_{5}(\kappa, d)\left(L^{4 d-2} e^{-L / d}+e^{-\frac{L}{d}}\right) \leq C_{5}(\kappa, d) L^{4 d} e^{-L / d} .
$$

If $L$ is sufficiently large, then this estimate implies the bound

$$
\mathscr{E}\left(u, \Pi_{2 d}\right) \leq e^{-L /(2 d)}
$$

Clearly, for all sufficiently large $L$ we have $[-L, L] \times[-1,+1]^{d-1} \subseteq \Pi_{2 d}$, and (4.21) contradicts the lower bound (4.14). We conclude that there is $L_{0}$ such that for all $L \geq L_{0}$, we have

$$
u(0, z) \geq \frac{2}{3}, \quad \forall z \in \mathbb{R}^{d-1}
$$

Our arguments also ensure that the constant $L_{0}$ depends only on $\kappa$ and $d$. This completes the proof.

Lemma 4.5 For any $p \geq 1$ there exists a constant $C_{0}$ that depends only on $p$, the dimension, the ellipticity constant $\kappa$ and the $L^{\infty}$ bound on $V$, such that for all $\lambda \leq 1$ and $t \geq 1 / \lambda^{2}$ and for any environment $\omega$,

$$
E_{0}^{\lambda, \omega}\left[\max _{0 \leq s \leq t}|X(s)|^{p}\right] \leq C_{0} \lambda^{p} t^{p}
$$

Proof

First assume that $V=0$. Letting $\widetilde{X}(t)=\lambda X\left(\frac{t}{\lambda^{2}}\right), t \geq 0$, we reduce (4.22) to the following inequality: for all $t \geq 1$ and for all $\lambda \leq 1$,

$$
E_{0}^{\lambda, \omega}\left[\max _{0 \leq s \leq t}|\widetilde{X}(s)|^{p}\right] \leq C_{0} t^{p} .
$$

Denote $\widetilde{T}_{r}=\inf \{s>0:|\widetilde{X}(s)|=r\}$.

We now rely on Aronson's lower bound for the Green function $\mathscr{G}(x, y, t)$ of the parabolic problem

$$
2 \partial_{t} U-\operatorname{div}(a(x) \nabla U)-a(x) e_{1} \cdot \nabla U=0,\left.\quad U\right|_{|x|=1}=0 .
$$

According to [1], Theorems 8 and 9 , for $t \leq 1$ and $x$ with $|x| \leq 1 / 2$ we have

$$
\mathscr{G}(x, y, t) \geq k t^{-d / 2} \exp \left(-K|x-y|^{2} / t\right)
$$


with constants $k>0$ and $K>0$ which only depend on $\kappa$ and $d$. This implies the bound

$$
P_{0}^{\lambda, \omega}\left[\widetilde{T}_{1} \geq 1\right] \geq \delta_{0}>0
$$

which, in turn, yields

$$
E_{0}^{\lambda, \omega}\left[e^{-\widetilde{T}_{1}}\right] \leq 1-\varepsilon_{0}
$$

for some $\varepsilon_{0}>0$ which only depends on $\kappa$ and $d$.

We have

$$
\begin{gathered}
E_{0}^{\lambda, \omega}\left[\max _{0 \leq s \leq t}|\widetilde{X}(s)|^{p}\right]=\int_{0}^{\infty} p r^{p-1} P_{0}^{\lambda, \omega}\left[\widetilde{T}_{r} \leq t\right] d r= \\
=\int_{0}^{\infty} p r^{p-1} P_{0}^{\lambda, \omega}\left[e^{-\widetilde{T}_{r}} \geq e^{-t}\right] d r \leq e^{t} \int_{0}^{\infty} p r^{p-1} E_{0}^{\lambda, \omega}\left[e^{-\widetilde{T}_{r}}\right] d r
\end{gathered}
$$

Considering the inequality

$$
\widetilde{T}_{r} \geq \widetilde{T}_{1}+\widetilde{T}_{1} \circ \theta_{\widetilde{T}_{1}}+\widetilde{T}_{1} \circ \theta_{\widetilde{T}_{2}}+\cdots+\widetilde{T}_{1} \circ \theta_{\widetilde{T}_{[r-1]}},
$$

by the Markov property and (4.24), for all $t \leq 1$ we have

$$
E_{0}^{\lambda, \omega}\left[\max _{0 \leq s \leq t}|\widetilde{X}(s)|^{p}\right] \leq e^{t} \int_{0}^{\infty} p r^{p-1} E_{0}^{\lambda, \omega}\left[e^{-\widetilde{T}_{r}}\right] d r \leq e \int_{0}^{\infty} p r^{p-1}\left(1-\varepsilon_{0}\right)^{\lfloor r\rfloor} d r \leq C(p, \kappa, d) .
$$

Using Jensen's inequality and the Markov property again, for all $t \geq 1$ we obtain

$$
\begin{gathered}
E_{0}^{\lambda, \omega}\left[\max _{0 \leq s \leq t}|\widetilde{X}(s)|^{p}\right] \leq \\
\leq E_{0}^{\lambda, \omega}\left[\left(\max _{0 \leq s \leq 1}|\widetilde{X}(s)|+\max _{1 \leq s \leq 2}|\widetilde{X}(s)-\widetilde{X}(1)|+\ldots+\max _{\lfloor t\rfloor \leq s \leq t}|\widetilde{X}(s)-\widetilde{X}(\lfloor t\rfloor)|\right)^{p}\right] \\
\leq(t+1)^{p-1}\left(E_{0}^{\lambda, \omega}\left[\max _{0 \leq s \leq 1}|\widetilde{X}(s)|^{p}\right] \quad+E_{0}^{\lambda, \omega}\left[\max _{1 \leq s \leq 2}|\widetilde{X}(s)-\widetilde{X}(1)|^{p}\right]\right. \\
\left.+\ldots+E_{0}^{\lambda, \omega}\left[\max _{\lfloor t\rfloor \leq s \leq t}|\widetilde{X}(s)-\widetilde{X}(\lfloor t\rfloor)|^{p}\right]\right) \\
\leq C(p, \kappa, d)(t+1)^{p} \leq C_{1}(p, \kappa, d) t^{p} .
\end{gathered}
$$

Recalling the definition of $\widetilde{X}$, we see that this is equivalent to (4.22). Hence the proof is complete in the case $V=0$.

To extend the statement to the case $V \neq 0$, we use the time change arguments from the last part of the proof of Proposition 3.1. We observe that as in the $V=0$ case, the process $Y^{\lambda, \omega}$ satisfies estimate (4.22), and due to (3.12), a similar bound also holds for the process $X^{\lambda, \omega}$. This completes the proof of the Lemma.

Lemma 4.5 implies the following bound on the effective drift:

$$
|\ell(\lambda)| \leq C_{0} \lambda
$$




\section{Renewal structure}

As already mentioned in the introduction, $X$ satisfies a Law of Large Numbers under $\mathbb{P}_{0}^{\lambda}$. The next Proposition is a quantitative version of this convergence.

\section{Proposition 5.1}

$$
\lim _{\alpha \rightarrow+\infty} \limsup _{\lambda \rightarrow 0 ; t \rightarrow+\infty ; \lambda^{2} t=\alpha}\left|\mathbb{E}_{0}^{\lambda}\left[\frac{X(t)}{\lambda t}\right]-\frac{\ell(\lambda)}{\lambda}\right|=0
$$

The proof of Proposition 5.1 is based on a renewal argument. More precisely, (5.1) follows if we can construct a renewal structure such that the interval between two successive renewal times is of order $1 / \lambda^{2}$.

Our definition of regeneration times is a variant of that in [25] where the construction depends on $\lambda$, whereas in [25] $\lambda$ was fixed.

We shall also heavily rely on the PDE estimates proved in the previous section of the paper.

The first issue we have to address is to check that the approach developed in [25] applies to our model and does yield the Law of Large Numbers. Besides in the proof of Proposition 5.1 we need sharp estimates on the regeneration times.

\subsection{Construction of regeneration times}

We recall that $\lambda$ is chosen small enough. In particular, we assume that $\frac{1}{\lambda}$ is much larger than the range $R$ in Assumption 4. We shall also need a constant $l \geq 1$ chosen so that $c e^{-\kappa_{1} l} \leq \frac{1}{2}$ where $c$ and $\kappa_{1}$ are the constants appearing in Lemma 4.1.

We set $R(\lambda):=\frac{l}{\lambda}$. We now follow the construction of [25], replacing $R$ in his construction with $R(\lambda)$. For details of this construction, proofs (and for pictures), we refer to [25]. We first have to enlarge the probability space by adding an auxiliary sequence $\left(Y_{k}\right)_{k \geq 0}$ of i.i.d. Bernoulli random variables. Denote $B_{R}(x)$ the ball with center $x$ and radius $R$. Let $U^{x}:=B_{6 R(\lambda)}\left(x+5 R(\lambda) e_{1}\right), B^{x}:=$ $B_{R(\lambda)}\left(x+9 R(\lambda) e_{1}\right)$, and let

$$
T_{\text {exit, } \mathrm{U}^{\mathrm{x}}}:=\inf \left\{s \geq 0: X(s) \notin U^{x}\right\}
$$

be the exit time from $U^{x}$. We consider the corresponding transition density $p_{\lambda, \omega, U^{x}}(s, x, y)$ which is

defined by $P_{x}^{\lambda, \omega}\left[X(s) \in G, T_{\text {exit, } \mathrm{U}^{\mathrm{x}}}>s\right]=\int_{G} p_{\lambda, \omega, U^{x}}(s, x, y) d y$, for all open sets $G \subseteq U^{x}$. We will need the following bound for this transition density.

Lemma 5.2 There is some $\delta>0$ (depending on $V, \sigma$ and $d$ ) such that

$$
p_{\lambda, \omega, U^{x}}\left(1 / \lambda^{2}, x, y\right) \geq \frac{2 \delta}{\left|B_{R(\lambda)}\right|}, \text { for all } x \in \mathbb{R}^{d}, y \in B^{x} \text { and } \lambda \leq 1 \text {. }
$$

Proof

Again we begin with the case $V=0$. After rescaling $t / \lambda^{2} \rightarrow t,(x / \lambda, y / \lambda) \rightarrow(x, y)$ the required bound is an immediate consequence of Theorems 8,9 in [1].

If $V \neq 0$, then the desired lower bound is an immediate consequence of the following statement: 
Lemma 5.3 Let a function $\rho(x)$ and a symmetric matrix $\left\{\alpha_{i j}(x)\right\}$ satisfy the estimates

$$
\varkappa \leq \rho \leq \varkappa^{-1}, \quad \varkappa I \leq \alpha \leq \varkappa^{-1} I, \quad \varkappa>0,
$$

and denote by $G_{B}(t, x, y)$ a solution to the following parabolic problem

$$
\begin{array}{r}
\rho(x) \partial_{t} G_{B}(t, x, y)-\operatorname{div}_{x}\left(\alpha(x) \nabla_{x} G_{B}(t, x, y)\right)=0, \\
\left.G_{B}\right|_{x \in \partial B}=0, \quad G_{B}(0, x, y)=\delta(x-y)
\end{array}
$$

with $B$ being an open ball centered at the origin, and $y \in B$. Then for any ball $B_{0}$ such that $\bar{B}_{0} \subset B$, the following inequality holds

$$
G_{B}(1, x, 0) \geq C \quad \text { for } x \in B_{0},
$$

with a constant $C$ which only depends on $\varkappa, d, B$ and $B_{0}$.

As in the case $V=0$ the estimate (5.3) can be obtained from (5.5) by scaling $t / \lambda^{2} \rightarrow t,(x / \lambda, y / \lambda) \rightarrow$ $(x, y)$.

Proof of Lemma 5.3. Enlarging if necessary the ball $B_{0}$ we assume without loss of generality, that $B_{0}$ contains the origin.

Consider an auxiliary spectral problem in $B$

$$
-\operatorname{div}(\alpha(x) \nabla \Psi(x))=\rho(x) \nu \Psi(x),\left.\quad \Psi\right|_{\partial B}=0 .
$$

By means of the minimax principle one can check that the principal eigenvalue $v_{1}$ satisfies the estimate $0<v_{1}<C_{1}$. The principal eigenfunction $\Psi_{1}$ is known to be positive in $B$. Assuming the normalization

$$
\int_{B} \Psi_{1}(x) d x=1
$$

by the Harnack inequality and Hölder continuity arguments (see [10]) we conclude that

$$
\Psi_{1}(x) \leq C_{2} \quad \text { in } B, \quad \Psi_{1}(x) \geq C_{3} \quad \text { in } B_{0},
$$

where the constant $C_{1}$ and $C_{2}$ depend only on $\varkappa, d$ and $B$, and $C_{3}$ also depends on $B_{0}$. Clearly, the function $e^{-v_{1} t} \Psi_{1}(x)$ solves problem (5.4) with the initial condition $\Psi_{1}$. Therefore,

$$
e^{-v_{1} / 2} \Psi_{1}(x)=\int_{B} G_{B}(1 / 2, x, y) \Psi_{1}(y) d y .
$$

Making use of (5.6) and the upper bound for $v_{1}$ we derive the inequality

$$
\int_{B} G_{B}(1 / 2,0, y) d y \geq C_{4}
$$

Considering the symmetry of the operator with respect to the weighted measure $\rho d x$, we have $\rho(x) G_{B}(t, x, y)=\rho(y) G_{B}(t, y, x)$. It readily follows from the results of [11] that the function $G(1 / 2, y, 0)$ satisfies the upper bound $G_{B}(1 / 2, y, 0) \leq C_{5}$ in $B$ with a constant $C_{5}$ which only depends on $\kappa$ and $d$. Consequently, there is a smaller ball $B_{1}$ centered at the origin, $\bar{B}_{1} \subset B$, such that

$$
\int_{B_{1}} G_{B}(1 / 2, y, 0) d y \geq C_{4} / 2
$$


the radius of $B_{1}$ depends only on $B, \kappa$ and $d$. This yields

$$
\sup _{y \in B_{1}} G_{B}(1 / 2, y, 0) \geq C_{6}
$$

Without loss of generality we suppose that $B_{0} \subset B_{1}$.

According to [11, Theorem 1-5], the function $G_{B}(1, y, 0)$ satisfies the following version of the Harnack inequality in $B_{1}$ :

$$
\inf _{y \in B_{1}} G_{B}(1, y, 0) \geq C_{7}(\varkappa, d) \sup _{y \in B_{1}} G_{B}(1 / 2, y, 0) .
$$

This estimate combined with (5.7) yields (5.5). This completes the proof of Lemmata 5.3 and 5.2.

Due to (5.3), we can give the following coupling construction. Let $\left(\mathscr{F}_{t}\right)_{t \geq 0}$ be the filtration generated by $(X(t))_{t \geq 0}$ and $\mathscr{S}_{m}:=\sigma\left(Y_{0}, \ldots, Y_{m}\right)$.

We denote $\theta_{m}^{\lambda}$ the rescaled shift operator defined by

$$
\theta_{m}^{\lambda}\left(\left(X(s)_{s \geq 0}\right)=\left(X\left(\lambda^{-2} m+s\right)\right)_{s \geq 0}\right.
$$

These shift operators $\theta_{m}^{\lambda}, m \in \mathbb{N}$, are extended in the obvious way: $\theta_{m}^{\lambda}\left((X(s))_{s \geq 0},\left(Y_{k}\right)_{k \geq 0}\right)=\left(\left(X\left(\lambda^{-2} m+\right.\right.\right.$ $\left.s))_{s \geq 0},\left(Y_{m+k}\right)_{k \geq 0}\right)$.

Proposition 5.4 There exists, for every $\lambda, \omega$ and $x$, a probability measure $\widehat{P}_{x}^{\lambda, \omega}$ on the enlarged probability space such that, with $\delta$ from (5.3),

(i) The law of $(X(t))_{t \geq 0}$ under $\widehat{P}_{x}^{\lambda, \omega}$ is $P_{x}^{\lambda, \omega}$, and the sequence $\left(Y_{k}\right)_{k \geq 0}$ is a sequence of i.i.d. Bernoulli variables with success probability $\delta$ under $\widehat{P}_{x}^{\lambda, \omega}$.

(ii) Under $\widehat{P}_{x}^{\lambda, \omega},\left(Y_{n}\right)_{n \geq m}$ is independent of $\mathscr{F}_{\lambda-2} \times \mathscr{S}_{m-1}$, and conditioned on $\mathscr{F}_{\lambda-2} \times \mathscr{S}_{m}, X \circ \theta_{m}^{\lambda}$ has the same law as $X$ under $\widehat{P}_{X\left(\lambda^{-2} m\right), Y_{m}}^{\lambda, \omega}$, where $\widehat{P}_{x, y}^{\lambda, \omega}$ denotes the conditioned law $\widehat{P}_{x}^{\lambda, \omega}\left[\cdot \mid Y_{0}=y\right]$, (for $y \in\{0,1\})$.

(iii) $\widehat{P}_{x, 1}^{\lambda, \omega}$-almost surely, $X(t) \in U^{x}$ for $t \in\left[0, \lambda^{-2}\right]$ and the distribution of $X\left(\lambda^{-2}\right)$ under $\widehat{P}_{x, 1}^{\lambda, \omega}$ is the uniform distribution on $B^{x}$.

We refer to [25] for the proof.

We will now introduce random times $N_{k} \in \lambda^{-2} \mathbb{Z}^{+}$for which $Y_{\lambda^{2} N_{k}}=1$ and for which the process $\left(e_{1} \cdot X(t)\right)_{t \geq 0}$ essentially reaches a local maximum (within a variation of $R(\lambda)$ ). The first regeneration time $\tau_{1}$ will be the first time $N_{k}+\lambda^{-2}, k \geq 1$ such that $\left(e_{1} \cdot X(t)\right)_{t \geq 0}$ never goes below $e_{1} \cdot X\left(N_{k}+\right.$ $\left.\lambda^{-2}\right)-R(\lambda)$ after $N_{k}+\lambda^{-2}$. In order to define $N_{k}$, we will first consider stopping times $\widetilde{N}_{k} \in \lambda^{-2} \mathbb{Z}^{+}$ which are essentially the times when $\left(e_{1} \cdot X(t)\right)_{t \geq 0}$ reaches local maxima (also within a variation of $R(\lambda)$ ). Then, $N_{1}$ will be the first $\widetilde{N}_{k}$ with $Y_{\lambda^{2} \widetilde{N}_{k}}=1$.

Let

$$
M(t):=\sup \left\{e_{1} \cdot(X(s)-X(0)): 0 \leq s \leq t\right\}
$$

For $a>0$, define the stopping times $V_{k}^{\lambda}(a), k \geq 1$, as follows. Recall that $T_{L}=\inf \left\{t: e_{1} \cdot X(t)=L\right\}$, and define

$$
V_{0}^{\lambda}(a):=T_{\lambda-1} a, \quad V_{k+1}^{\lambda}(a):=T_{M\left(\left\lceil V_{k}^{\lambda}(a)\right\rceil_{\lambda}\right)+R(\lambda)}, \quad k \geq 1
$$


here and later on $\lceil r\rceil_{\lambda}$ stands for the $\min \left\{n \in \lambda^{-2} \mathbb{Z}: r \leq n\right\}$. Then

$$
\begin{gathered}
\widetilde{N}_{1}^{\lambda}(a):=\inf \left\{\left\lceil V_{k}^{\lambda}(a)\right\rceil_{\lambda}: k \geq 0, \sup _{s \in\left[V_{k}^{\lambda}(a),\left\lceil V_{k}^{\lambda}(a)\right]_{\lambda}\right]}\left|e_{1} \cdot\left(X(s)-X\left(V_{k}^{\lambda}(a)\right)\right)\right| \leq \frac{R(\lambda)}{2}\right\}, \\
\widetilde{N}_{k+1}^{\lambda}(a):=\widetilde{N}_{1}^{\lambda}(3 \lambda R(\lambda)) \circ \theta_{\lambda^{2} \widetilde{N}_{k}^{\lambda}(a)}^{\lambda}+\widetilde{N}_{k}^{\lambda}(a), \quad k \geq 1, \\
N_{1}^{\lambda}(a):=\inf \left\{\widetilde{N}_{k}^{\lambda}(a): k \geq 1, Y_{\lambda^{2} \widetilde{N}_{k}^{\lambda}(a)}=1\right\},
\end{gathered}
$$

(we will see later that $\widetilde{N}_{k}^{\lambda}(a)<\infty$, for all $k$ ). The random times $\lambda^{2} \widetilde{N}_{k}^{\lambda}(a)$ are integer-valued and $\sup _{\widetilde{N}_{k}} e_{1} \cdot\left(X(s)-X\left(\widetilde{N}_{k}^{\lambda}(a)\right)\right) \leq R(\lambda)$. We next define random times $S_{1}, J_{1}$ and $R_{1}$ as follows. $s \leq \tilde{N}_{k}^{\lambda}(a)$

$$
S_{1}^{\lambda}:=N_{1}^{\lambda}(3 \lambda R(\lambda))+\lambda^{-2}, \quad J_{1}^{\lambda}:=S_{1}^{\lambda}+T_{-R(\lambda)} \circ \theta_{\lambda^{2} S_{1}^{\lambda}}^{\lambda}, \quad R_{1}^{\lambda}:=\left\lceil J_{1}^{\lambda}\right\rceil_{\lambda}=S_{1}^{\lambda}+D \circ \theta_{\lambda^{2} S_{1}^{\lambda}}^{\lambda},
$$

where

$$
D:=\left\lceil T_{-R(\lambda)}\right\rceil \lambda
$$

Now we proceed recursively:

$$
N_{k+1}^{\lambda}=R_{k}^{\lambda}+N_{1}^{\lambda}\left(a_{k}\right) \circ \theta_{\lambda^{2} R_{k}^{\lambda}}^{\lambda} \quad \text { with } a_{k}=\lambda\left(M\left(R_{k}^{\lambda}\right)-e_{1} \cdot\left(X\left(R_{k}^{\lambda}\right)-X(0)\right)+R(\lambda)\right)
$$

and

$$
S_{k+1}^{\lambda}:=N_{k+1}^{\lambda}+\lambda^{-2}, \quad J_{k+1}^{\lambda}:=S_{k+1}^{\lambda}+T_{R(\lambda)} \circ \theta_{\lambda^{2} S_{k+1}^{\lambda}}^{\lambda}, \quad R_{k+1}^{\lambda}:=\left\lceil J_{k+1}^{\lambda}\right\rceil \lambda=S_{k+1}^{\lambda}+D \circ \theta_{\lambda^{2} S_{k+1}^{\lambda}}^{\lambda} .
$$

Note that for all $k$, the $\mathscr{F}_{t} \times \mathscr{S}_{\lambda^{2}\lceil t\rceil_{\lambda}}$ - stopping times $\lambda^{2} N_{k}^{\lambda}, \lambda^{2} S_{k}^{\lambda}$ and $\lambda^{2} R_{k}^{\lambda}$ are integer-valued (the value $+\infty$ is possible). By definition, we have $\lambda^{-2} \leq N_{1}^{\lambda} \leq S_{1}^{\lambda} \leq J_{1}^{\lambda} \leq R_{1}^{\lambda} \leq N_{2}^{\lambda} \leq S_{2}^{\lambda} \leq J_{2}^{\lambda} \leq R_{2}^{\lambda} \leq$ $N_{3}^{\lambda} \ldots \leq \infty$. The first regeneration time $\tau_{1}$ is defined as

$$
\tau_{1}:=\inf \left\{S_{k}^{\lambda}: S_{k}^{\lambda}<\infty, R_{k}^{\lambda}=\infty\right\} \leq \infty
$$

By definition, $\lambda^{2} \tau_{1}$ is integer-valued and $\tau_{1} \geq 2 \lambda^{-2}$ (since $N_{1}^{\lambda} \geq \lambda^{-2}$ ). We see that on the event $\tau_{1}<\infty$ it holds

$$
e_{1} \cdot X(s) \leq e_{1} \cdot X\left(\tau_{1}-\lambda^{-2}\right)+R(\lambda) \leq e_{1} \cdot X\left(\tau_{1}\right)-7 R(\lambda), \quad \text { for } s \leq \tau_{1}-\lambda^{-2}, \widehat{P}_{x}^{\lambda, \omega}-\text { a.s. },
$$

see also Proposition 5.4, i.e. $(X(s))_{s \leq \tau_{1}-\lambda^{-2}}$ remains in the halfspace $\left\{z \in \mathbb{R}^{d}: e_{1} \cdot z \leq e_{1} \cdot X\left(\tau_{1}\right)-\right.$ $7 R(\lambda)\}$. On the other hand, since the process $\left(e_{1} \cdot X(t)\right)_{t \geq 0}$ never goes below $e_{1} \cdot X\left(\tau_{1}\right)-R(\lambda)$ after $\tau_{1}, \widehat{P}_{x}^{\lambda, \omega}$-a.s., $(X(t))_{t>\tau_{1}}$ remains in the halfspace $\left\{z \in \mathbb{R}^{d}: e_{1} \cdot z \geq e_{1} \cdot X\left(\tau_{1}\right)-R(\lambda)\right\}$.

In [25], it is proved that $\tau_{1}<\infty$ if and only if the process is transient in direction $e_{1}$. More precisely, define the annealed law

$$
\widehat{\mathbb{P}}_{x}^{\lambda}[A]:=\int d \mathbb{Q}(\omega) \int d \widehat{P}_{x}^{\lambda, \omega}(w) \mathbf{1}_{A}(\omega, w)
$$


Proposition 5.5 The following statements are equivalent:

(i) $\tau_{1}<\infty, \widehat{\mathbb{P}}_{0}^{\lambda}$-a.s.

(ii) $e_{1} \cdot X(t) \rightarrow \infty, \mathbb{P}_{0}^{\lambda}$-a.s.

(iii) $\mathbb{P}_{0}^{\lambda}[D=\infty]>0$.

For the proof, we refer to [25], Lemma 2.3 and Proposition 2.7. Later on we will need stronger results than those of Proposition 5.5 involving sharp bounds on $\tau_{1}$ as $\lambda \rightarrow 0$.

We set $\tau_{0}=0$ for convenience. The next theorem (Theorem 2.5 in [25]) gives the renewal structure which is crucial to establish (for fixed values of $\lambda$ ) the Law of Large Numbers and the Central Limit Theorem.

Theorem 5.6 Assume that $\tau_{1}<\infty, \widehat{\mathbb{P}}_{0}^{\lambda}$-a.s. Then, under the measure $\widehat{\mathbb{P}}_{0}^{\lambda}$, the random variables $Z_{k}:=\left(\left(X\left(\left(\tau_{k}+t\right) \wedge\left(\tau_{k+1}-\lambda^{-2}\right)\right)-X\left(\tau_{k}\right)\right)_{t \geq 0}, X\left(\tau_{k+1}\right)-X\left(\tau_{k}\right), \tau_{k+1}-\tau_{k}\right), k \geq 0$ are independent. Furthermore, the random variables $Z_{k}, k \geq 1$, are i.i.d. under $\widehat{\mathbb{P}}_{0}^{\lambda}$ and have the same law as $Z_{0}$ under $\widehat{\mathbb{P}}_{0}^{\lambda}[\cdot \mid D=\infty]$.

Note that the renewal structure is proved for the trajectory between the times $\tau_{k}$ and $\tau_{k+1}-\lambda^{-2}$, but we have a good control over the trajectory between the times $\tau_{k+1}-\lambda^{-2}$ and $\tau_{k+1}$ : since $Y_{\lambda^{2} \tau_{k+1}-1}=1$, then $X(s) \in U^{X_{\tau_{k+1}-1 / \lambda^{2}}}$, for all $s \in\left[\tau_{k+1}-\lambda^{-2}, \tau_{k+1}\right]$, i.e. the trajectory remains in a ball of radius $6 R(\lambda)$.

Let

$$
K=\inf \left\{k \geq 1: S_{k}^{\lambda}<\infty \text { and } R_{k}^{\lambda}=\infty\right\}
$$

Then $\tau_{1}=S_{K}^{\lambda}$. The points $X\left(S_{1}^{\lambda}\right), X\left(S_{2}^{\lambda}\right), \ldots$ are ladder points of the process. The idea of such a decomposition of the path goes back to [14] and was first turned effective for multi-dimensional random walks in random environments in [28].

\section{Lemma 5.7 The following statements hold:}

(i) $S_{1}^{\lambda}$ is $\widehat{\mathbb{P}}_{0}^{\lambda}$-almost surely finite and, for all $k$, $S_{k}^{\lambda}$ is $\widehat{\mathbb{P}}_{0}^{\lambda}$-almost surely finite on the event $R_{k-1}^{\lambda}<\infty$.

(ii) $\tau_{k}$ is $\widehat{\mathbb{P}}_{0}^{\lambda}$ almost surely finite for all $k \geq 1$.

(iii) $\widehat{\mathbb{E}}_{0}^{\lambda}\left[\tau_{k}\right]<\infty$ for all $k \geq 1$.

Proof Part (i). Due to Lemma 4.2, $V_{k}^{\lambda}(a)<\infty, \widehat{\mathbb{P}}_{x}^{\lambda}$-a.s for all $k$ and all $x$. We have (as in Lemma 5.2 this is a consequence of the Aronson-Nash lower bounds for the Green function of a parabolic equation)

$$
\inf _{\lambda \leq 1} \inf _{x \in \mathbb{R}^{d}} \inf _{\omega} \widehat{P}_{x}^{\lambda, \omega}\left[\max _{s \leq 1 / \lambda^{2}}\left|e_{1} \cdot X(s)\right| \leq \frac{R(\lambda)}{2}\right]=\widetilde{\delta}>0,
$$

and this implies that $\widetilde{N}_{k}^{\lambda}(a)<\infty$ for all $a, k, \widehat{\mathbb{P}}_{x}^{\lambda}$-a.s for all $x$. Due to Proposition 5.4, we conclude that $N_{k}^{\lambda}(a)<\infty$ for all $a, k, \widehat{\mathbb{P}}_{x}^{\lambda}$-a.s for all $x$.

Part (ii). It follows from Lemma 4.1 that under proper choice of $l$ in the definition of $R(\lambda)$, the following bound holds

$$
\widehat{\mathbb{P}}_{0}^{\lambda}[K=1]=\widehat{\mathbb{P}}_{0}^{\lambda}\left[R_{1}^{\lambda}=\infty\right] \geq 1-c e^{-\kappa_{1} l} \geq \frac{1}{2}
$$


Lemma 4.1 and the Markov property applied at time $S_{k+1}^{\lambda}$ also imply that

$$
\widehat{\mathbb{P}}_{0}^{\lambda}\left[R_{k+1}^{\lambda}=\infty \mid R_{k}^{\lambda}<\infty\right] \geq \frac{1}{2}
$$

Thus we get that

$$
\widehat{\mathbb{P}}_{0}^{\lambda}[K \geq k] \leq 2^{-k+1}
$$

and

$$
\widehat{\mathbb{P}}_{0}^{\lambda}[K=\infty]=0 .
$$

Together with part (i), this implies part (ii).

We now turn to part (iii). The next lemma gives a bound for the tail of the random variable $\tau_{1}$, which will be sufficient to guarantee that $\tau_{1}$ has finite expectation under $\widehat{\mathbb{P}}_{0}^{\lambda}$.

Lemma 5.8 There exist constants $c_{3}>0$ and $c_{4}>0$ such that

$$
\text { for all } \lambda \leq 1 \text { and } t>0, \quad \widehat{\mathbb{P}}_{0}^{\lambda}\left[\tau_{1} \geq \lambda^{-2} t\right] \leq c_{3} e^{-c_{4} t}
$$

The same tail bound holds for the differences $\tau_{k+1}-\tau_{k}$ for all $k \geq 1$ :

$$
\text { for all } \lambda \leq 1 \text { and } t>0, \quad \widehat{\mathbb{P}}_{0}^{\lambda}\left[\tau_{k+1}-\tau_{k} \geq \lambda^{-2} t\right] \leq c_{3} e^{-c_{4} t} \text {. }
$$

Proof of Lemma 5.8. It suffices to show (5.22). Then, (5.23) follows since

$$
\widehat{\mathbb{P}}_{0}^{\lambda}\left[\tau_{k+1}-\tau_{k} \geq \lambda^{-2} t\right]=\widehat{\mathbb{P}}_{0}^{\lambda}\left[\tau_{1} \geq \lambda^{-2} t \mid D=\infty\right]
$$

(see Theorem 5.6) and $\widehat{\mathbb{P}}_{0}^{\lambda}[D=\infty] \geq \frac{1}{2}$, see (5.20). To show (5.22), we claim that the following stronger statement holds: There exist constants $c_{3}>0$ and $c_{4}>0$ such that for all $\lambda \leq 1, t>0$ and all $\omega$,

$$
\widehat{P}_{0}^{\lambda, \omega}\left[\tau_{1} \geq \lambda^{-2} t\right] \leq c_{3} e^{-c_{4} t} .
$$

Since one can follow the proof of Corollary 4.10 in [25], we give only a sketch of the proof of (5.24). Step 1 (corresponds to Proposition 4.7 in [25]). Recall (5.8). There exists a constant $c_{5}>0$ such that

$$
\sup _{\omega} \widehat{E}_{0}^{\lambda, \omega}\left[\exp \left(c_{5} \lambda e_{1} \cdot M\left(T_{-R(\lambda)}\right)\right) \mathbf{1}\left(T_{-R(\lambda)}<\infty\right)\right]<\infty .
$$

Proof of (5.25): Due to Lemma 4.1, $\widehat{P}_{0}^{\lambda, \omega}\left[\lambda e_{1} \cdot M\left(T_{-R(\lambda)}\right)>t, T_{-R(\lambda)}<\infty\right] \leq c e^{-\kappa_{1}(l+t)}$ and this implies (5.25).

Step 2 Follow the proof of Theorem 4.9 in [25] to obtain that there is a constant $c_{6}>0$ such that for all $\lambda \leq 1$,

$$
\sup _{\omega} \widehat{E}_{0}^{\lambda, \omega}\left[\exp \left(c_{6} \lambda e_{1} \cdot X_{\tau_{1}}\right)\right]<\infty .
$$

Step 3 Take $t>\frac{6 l}{\kappa_{2}}$ and $u=\frac{\kappa_{2}}{2} t$ where $\kappa_{2}$ is the constant from Lemma 4.2. Then,

$$
\widehat{P}_{0}^{\lambda, \omega}\left[\tau_{1} \geq \lambda^{-2} t\right] \leq \widehat{P}_{0}^{\lambda, \omega}\left[\tau_{1} \geq \lambda^{-2} t, e_{1} \cdot X_{\tau_{1}}<\lambda^{-1} u-3 R(\lambda)\right]+\widehat{P}_{0}^{\lambda, \omega}\left[e_{1} \cdot X_{\tau_{1}} \geq \lambda^{-1} u-3 R(\lambda)\right]
$$


Due to Step 2, the second term on the r.h.s. of (5.27) is $\leq e^{-c_{7} u}$ for some $c_{7}>0$. Turning to the first term in (5.27), note that since

$$
\sup _{s \leq \tau_{1}} e_{1} \cdot\left(X_{s}-X_{\tau_{1}}\right)<3 R(\lambda)
$$

see the definition of $U^{x}$ and $B^{x}$ before (5.2), $\widehat{P}_{0}^{\lambda, \omega}\left[\tau_{1} \geq \lambda^{-2} t, e_{1} \cdot X_{\tau_{1}}<\lambda^{-1} u-3 R(\lambda)\right] \leq P_{0}^{\lambda, \omega}\left[T_{\lambda-1} u\right.$ $\left.\lambda^{-2} t\right] \leq C e^{-\kappa_{2} t+u}$, where the last inequality comes from Lemma 4.2.

As mentioned before, the regeneration structure implies a law of large numbers for fixed $\lambda$.

Proposition 5.9 We have, for each $\lambda>0$,

$$
\lim _{t \rightarrow \infty} \frac{X(t)}{t}=\frac{\widehat{\mathbb{E}}_{0}^{\lambda}\left[X\left(\tau_{2}\right)-X\left(\tau_{1}\right)\right]}{\widehat{\mathbb{E}}_{0}^{\lambda}\left[\tau_{2}-\tau_{1}\right]} \quad \mathbb{P}_{0}^{\lambda} \text { - a.s. }
$$

As a consequence,

$$
\widehat{\mathbb{E}}_{0}^{\lambda}\left[X\left(\tau_{2}\right)-X\left(\tau_{1}\right)\right]=\widehat{\mathbb{E}}_{0}^{\lambda}\left[\tau_{2}-\tau_{1}\right] \ell(\lambda)
$$

Proof

Theorem 5.6 implies, with the ergodic Theorem, that if $\widehat{\mathbb{E}}_{0}^{\lambda}\left[\tau_{2}-\tau_{1}\right]$ is finite, then $\lim _{t \rightarrow \infty} \frac{X(t)}{t}$ exists, $\mathbb{P}_{0}^{\lambda}$-a.s. and (5.28) holds true. Once again, we refer to [25] for the details.

\subsection{Estimates on the regeneration times}

We now show that under $\widehat{P}_{0}^{\lambda}, \tau_{1}$ and $\tau_{2}-\tau_{1}$ are of order $\lambda^{-2}$. More precisely,

Lemma 5.10 We have

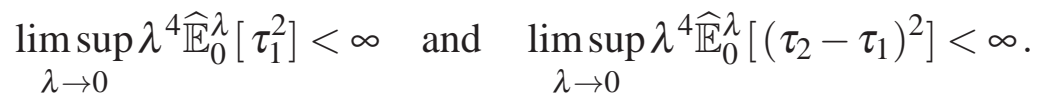

As a consequence,

$$
\underset{\lambda \rightarrow 0}{\limsup } \lambda^{2} \widehat{\mathbb{E}}_{0}^{\lambda}\left[\tau_{1}\right]<\infty \text { and } \limsup _{\lambda \rightarrow 0} \lambda^{2} \widehat{\mathbb{E}}_{0}^{\lambda}\left[\tau_{2}-\tau_{1}\right]<\infty,
$$

and

$$
\limsup _{\lambda \rightarrow 0} \frac{\widehat{\mathbb{E}}_{0}^{\lambda}\left[\tau_{1}^{2}\right]}{\widehat{\mathbb{E}}_{0}^{\lambda}\left[\tau_{1}\right]^{2}}<\infty \quad \text { and } \quad \limsup _{\lambda \rightarrow 0} \frac{\widehat{\mathbb{E}}_{0}^{\lambda}\left[\left(\tau_{2}-\tau_{1}\right)^{2}\right]}{\widehat{\mathbb{E}}_{0}^{\lambda}\left[\tau_{2}-\tau_{1}\right]^{2}}<\infty
$$

Proof

Observe that (5.31) directly follows from (5.30) and Hölder's inequality.

To deduce (5.32) from (5.30) it suffices to prove a lower bound on $\widehat{\mathbb{E}}_{0}^{\lambda}\left[\tau_{1}\right]$ (or $\widehat{\mathbb{E}}_{0}^{\lambda}\left[\tau_{2}-\tau_{1}\right]$, respectively) of the order $\lambda^{-2}$. But note that, since $\tau_{1} \geq T_{l / \lambda}$, we have

$$
\lambda^{2} \widehat{\mathbb{E}}_{0}^{\lambda}\left[\tau_{1}\right] \geq \widehat{\mathbb{P}}_{0}^{\lambda}\left[\tau_{1} \geq \lambda^{-2}\right] \geq \mathbb{P}_{0}^{\lambda}\left[T_{l / \lambda} \geq \lambda^{-2}\right]
$$

We next use the Girsanov formula (3.4):

$$
\mathbb{P}_{0}^{\lambda}\left[T_{l / \lambda} \geq \lambda^{-2}\right]=\int E\left[\mathbf{1}\left(T_{l / \lambda} \geq \lambda^{-2}\right) e^{\lambda \bar{B}\left(\frac{1}{\lambda^{2}}\right)-\frac{\lambda^{2}}{2}\langle\bar{B}\rangle\left(\frac{1}{\lambda^{2}}\right)}\right] d \mathbb{Q}
$$


Thus the invariance principle implies that this last quantity has a positive limit: namely it converges to $E\left[\mathbf{1}\left(T_{l} \geq 1\right) e^{Z-\frac{1}{2} E\left(Z^{2}\right)}\right]$, where $Z$ is some Gaussian random variable and $T_{l}$ is the hitting time of level $l$ by some Brownian motion. This last expectation cannot be 0 . The same argument applies to $\tau_{2}-\tau_{1}$.

Proof of (5.30): due to (5.22), we have $\widehat{\mathbb{P}}_{0}^{\lambda}\left[\tau_{1}^{2} \geq t \lambda^{-4}\right]=\widehat{\mathbb{P}}_{0}^{\lambda}\left[\tau_{1} \geq \sqrt{t} \lambda^{-2}\right] \leq c_{3} e^{-c_{4} \sqrt{t}}$. In the same way, due to (5.23), we have $\widehat{\mathbb{P}}_{0}^{\lambda}\left[\left(\tau_{2}-\tau_{1}\right)^{2} \geq t \lambda^{-4}\right] \leq c_{3} e^{-c_{4} \sqrt{t}}$, and (5.30) follows.

We can now show the corresponding bounds for the regeneration distances.

Lemma 5.11 We have

$$
\limsup _{\lambda \rightarrow 0} \lambda^{2} \widehat{\mathbb{E}}_{0}^{\lambda}\left[\left|X\left(\tau_{1}\right)\right|^{2}\right]<\infty
$$

and

$$
\limsup _{\lambda \rightarrow 0} \lambda^{2} \widehat{\mathbb{E}}_{0}^{\lambda}\left[\left|X\left(\tau_{2}\right)-X\left(\tau_{1}\right)\right|^{2}\right]<\infty
$$

We note for further reference that, as a consequence of (5.33) and (5.34),

$$
\sup _{k} \frac{1}{k^{2}} \limsup _{\lambda \rightarrow 0} \lambda^{2} \widehat{\mathbb{E}}_{0}^{\lambda}\left[\left|X\left(\tau_{k}\right)\right|^{2}\right]<\infty
$$

Moreover, note that due to (4.25),

$$
\limsup _{\lambda \rightarrow 0} \frac{|\ell(\lambda)|}{\lambda}<\infty .
$$

(This also follows from (5.34) together with (5.29), (5.31) and (5.32)).

Proof of Lemma 5.11 To show (5.33), note that

$$
\begin{aligned}
\widehat{\mathbb{E}}_{0}^{\lambda}\left[X\left(\tau_{1}\right)^{2}\right] & =\sum_{k=0}^{\infty} \widehat{\mathbb{E}}_{0}^{\lambda}\left[X\left(\tau_{1}\right)^{2} \mathbf{1}\left(k \leq \lambda^{2} \tau_{1}<k+1\right)\right] \\
& \leq \sum_{k=0}^{\infty} \widehat{\mathbb{E}}_{0}^{\lambda}\left[\max _{t \leq(k+1) / \lambda^{2}}|X(t)|^{2} \mathbf{1}\left(k \leq \lambda^{2} \tau_{1}\right)\right] \\
& \leq 2 \sum_{k=0}^{\infty}\left(\mathbb{E}_{0}^{\lambda}\left[\max _{t \leq(k+1) / \lambda^{2}}|X(t)|^{4}\right]\right)^{1 / 2}\left(\widehat{\mathbb{P}}_{0}^{\lambda}\left[\lambda^{2} \tau_{1} \geq k\right]\right)^{1 / 2} \\
& \leq 2 \sum_{k=0}^{\infty}\left(\lambda \frac{k+1}{\lambda^{2}}\right)^{2}\left(c_{3} e^{-c_{4} k}\right)^{1 / 2} \leq c_{8} \lambda^{-2}
\end{aligned}
$$

for some constant $c_{8}>0$, where we used (4.22) and (5.22). Now, (5.34) follows from (5.33), since

$$
\widehat{\mathbb{E}}_{0}^{\lambda}\left[\left|X\left(\tau_{2}\right)-X\left(\tau_{1}\right)\right|^{2}\right]=\widehat{\mathbb{E}}_{0}^{\lambda}\left[\left|X\left(\tau_{1}\right)\right|^{2} \mid D=\infty\right]
$$

(see Theorem 5.6) and $\widehat{\mathbb{P}}_{0}^{\lambda}[D=\infty] \geq \frac{1}{2}$, for all $\lambda$, see (5.20). 


\subsection{Proof of Proposition 5.1}

Let

$$
n(t):=\left\lfloor\frac{t}{\widehat{\mathbb{E}}_{0}^{\lambda}\left[\tau_{2}-\tau_{1}\right]}\right\rfloor
$$

We write

$$
X(t)=X\left(\tau_{n(t)}\right)+\left(X(t)-X\left(\tau_{n(t)}\right) .\right.
$$

We will show that

$$
\lim _{\alpha \rightarrow+\infty} \limsup _{\lambda \rightarrow 0 ; t \rightarrow+\infty ; \lambda^{2} t=\alpha}\left|\frac{1}{\lambda t} \widehat{\mathbb{E}}_{0}^{\lambda}\left[X\left(\tau_{n(t)}\right)\right]-\frac{\ell(\lambda)}{\lambda}\right|=0
$$

and that

$$
\lim _{\alpha \rightarrow+\infty} \limsup _{\lambda \rightarrow 0 ; t \rightarrow+\infty ; \lambda^{2} t=\alpha}\left|\frac{1}{\lambda t} \widehat{\mathbb{E}}_{0}^{\lambda}\left[X(t)-X\left(\tau_{n(t)}\right)\right]\right|=0 .
$$

(5.38) and (5.39) then imply

$$
\lim _{\alpha \rightarrow+\infty} \limsup _{\lambda \rightarrow 0 ; t \rightarrow+\infty ; \lambda^{2} t=\alpha}\left|\mathbb{E}_{0}^{\lambda}\left[\frac{X(t)}{\lambda t}\right]-\frac{\ell(\lambda)}{\lambda}\right|=0,
$$

i.e. Proposition 5.1.

To show (5.38), note that (recalling $\tau_{0}=0$ ),

$$
\left|\frac{1}{\lambda t} \widehat{\mathbb{E}}_{0}^{\lambda}\left[X\left(\tau_{n(t)}\right)\right]-\frac{\ell(\lambda)}{\lambda}\right|=\left|\frac{1}{\lambda t} \widehat{\mathbb{E}}_{0}^{\lambda}\left[\sum_{k=1}^{n(t)}\left(X\left(\tau_{k}\right)-X\left(\tau_{k-1}\right)\right)\right]-\frac{\ell(\lambda)}{\lambda}\right|
$$

Using formula (5.29) in Proposition 5.9, we rewrite and estimate the r.h.s. of (5.41) as

$$
\begin{gathered}
\left|\frac{1}{\lambda t} \widehat{\mathbb{E}}_{0}^{\lambda}\left[X\left(\tau_{1}\right)\right]+\frac{1}{\lambda t}(n(t)-1) \widehat{\mathbb{E}}_{0}^{\lambda}\left[X\left(\tau_{2}\right)-X\left(\tau_{1}\right)\right]-\frac{\ell(\lambda)}{\lambda}\right| \\
\leq\left|\frac{1}{\lambda t} \widehat{\mathbb{E}}_{0}^{\lambda}\left[X\left(\tau_{1}\right)\right]\right|+\left|\frac{\ell(\lambda)}{\lambda}\left(\frac{\widehat{\mathbb{E}}_{0}^{\lambda}\left[\tau_{2}-\tau_{1}\right]}{t}\left(\left\lfloor\frac{t}{\widehat{\mathbb{E}}_{0}^{\lambda}\left[\tau_{2}-\tau_{1}\right]}\right\rfloor-1\right)-1\right)\right| .
\end{gathered}
$$

Due to (5.33), the first term in the right hand side of (5.42) is of order $\alpha^{-1}$ and therefore satisfies

$$
\lim _{\alpha \rightarrow+\infty} \limsup _{\lambda \rightarrow 0 ; t \rightarrow+\infty ; \lambda^{2} t=\alpha}\left|\frac{1}{\lambda t} \widehat{\mathbb{E}}_{0}^{\lambda}\left[X\left(\tau_{1}\right)\right]\right|=0 .
$$

Now consider the second term in (5.42). ¿From (5.31), we know that $\widehat{\mathbb{E}}_{0}^{\lambda}\left[\tau_{2}-\tau_{1}\right] / t$ is of order $\alpha^{-1}$. Therefore

$$
\lim _{\alpha \rightarrow+\infty} \limsup _{\lambda \rightarrow 0 ; t \rightarrow+\infty ; \lambda^{2} t=\alpha}\left(\frac{\widehat{\mathbb{E}}_{0}^{\lambda}\left[\tau_{2}-\tau_{1}\right]}{t}\left(\left\lfloor\frac{t}{\widehat{\mathbb{E}}_{0}^{\lambda}\left[\tau_{2}-\tau_{1}\right]}\right\rfloor-1\right)-1\right)=0 .
$$

We also proved in (5.36) that $\frac{|\ell(\lambda)|}{\lambda}$ remains bounded for $\lambda$ tending to 0 . Hence we see that the second term in the right hand side of (5.42) also tends to 0 , thus proving that (5.38) holds true.

To show (5.39), we need the following lemma, whose proof is deferred. 
Lemma 5.12 We have, for each $\varepsilon>0$,

$$
\lim _{\alpha \rightarrow+\infty} \limsup _{\lambda \rightarrow 0 ; t \rightarrow+\infty ; \lambda^{2} t=\alpha} \widehat{\mathbb{P}}_{0}^{\lambda}\left[\left|\tau_{n(t)}-t\right| \geq \varepsilon t\right]=0 .
$$

We will split the integration in (5.39) according to the partition in the two events $\left\{\left|\tau_{n(t)}-t\right| \geq \varepsilon t\right\}$ and $\left\{(1-\varepsilon) t<\tau_{n(t)}<(1+\varepsilon) t\right\}$. We will show that, for each $\varepsilon>0$,

$$
\lim _{\alpha \rightarrow+\infty} \limsup _{\lambda \rightarrow 0 ; t \rightarrow+\infty ; \lambda^{2} t=\alpha} \mid \frac{1}{\lambda t} \widehat{\mathbb{E}}_{0}^{\lambda}\left[\left(X(t)-X\left(\tau_{n(t)}\right) \mathbf{1}\left(\left|\tau_{n(t)}-t\right| \geq \varepsilon t\right)\right] \mid=0,\right.
$$

and that

$$
\lim _{\varepsilon \rightarrow 0} \limsup _{\alpha \rightarrow+\infty} \limsup _{\lambda \rightarrow 0 ; t \rightarrow+\infty ; \lambda^{2} t=\alpha} \mid \frac{1}{\lambda t} \widehat{\mathbb{E}}_{0}^{\lambda}\left[\left(X(t)-X\left(\tau_{n(t)}\right) \mathbf{1}\left((1-\varepsilon) t<\tau_{n(t)}<(1+\varepsilon) t\right)\right] \mid=0 .\right.
$$

To show (5.44), we use Hölder's inequality to get that

$$
\begin{gathered}
\mid \widehat{\mathbb{E}}_{0}^{\lambda}\left[\left(X(t)-X\left(\tau_{n(t)}\right) \mathbf{1}\left(\left|\tau_{n(t)}-t\right| \geq \varepsilon t\right)\right] \mid\right. \\
\leq \widehat{\mathbb{E}}_{0}^{\lambda}\left[\mid X(t)-X\left(\left.\tau_{n(t)}\right|^{2}\right]^{1 / 2} \widehat{\mathbb{P}}_{0}^{\lambda}\left[\left|\tau_{n(t)}-t\right| \geq \varepsilon t\right]^{1 / 2}\right. \\
\leq 2\left(\widehat{\mathbb{E}}_{0}^{\lambda}\left[|X(t)|^{2}\right]+\widehat{\mathbb{E}}_{0}^{\lambda}\left[\left|X\left(\tau_{n(t)}\right)\right|^{2}\right]\right)^{1 / 2} \widehat{\mathbb{P}}_{0}^{\lambda}\left[\left|\tau_{n(t)}-t\right| \geq \varepsilon t\right]^{1 / 2},
\end{gathered}
$$

(for the last inequality, we used the formula $(x-y)^{2} \leq 2\left(x^{2}+y^{2}\right)$ ). Now, we conclude with (3.2), (5.35) and Lemma 5.12.

For (5.45), note that

$$
\begin{gathered}
\mid \widehat{\mathbb{E}}_{0}^{\lambda}\left[\left(X(t)-X\left(\tau_{n(t)}\right) \mathbf{1}\left((1-\varepsilon) t<\tau_{n(t)}<(1+\varepsilon) t\right)\right] \mid\right. \\
\leq \mathbb{E}_{0}^{\lambda}\left[\max _{(1-\varepsilon) t \leq u<s \leq(1+\varepsilon) t}|X(u)-X(s)|\right] \leq 2 \mathbb{E}_{0}^{\lambda}\left[\max _{(1-\varepsilon) t \leq u \leq(1+\varepsilon) t}|X(u)-X((1-\varepsilon) t)|\right] .
\end{gathered}
$$

Using the Markov property, we see that the last term equals

$$
g(\lambda, \varepsilon, t):=2 \mathbb{E}_{0}^{\lambda}\left[\max _{u \leq 2 \varepsilon t}|X(u)|\right] .
$$

Due to (3.2), for each $\varepsilon$,

$$
\sup _{\alpha \varepsilon \geq 1} \limsup _{\lambda \rightarrow 0 ; t \rightarrow+\infty ; \lambda^{2} \varepsilon t=\alpha} \frac{g(\lambda, \varepsilon, t)}{\lambda \varepsilon t}<\infty
$$

and this proves (5.45).

It remains to prove Lemma 5.12. We first show

Lemma 5.13 For all $\varepsilon>0$,

$$
\widehat{\mathbb{P}}_{0}^{\lambda}\left[\left|\tau_{k}-k \mathbb{E}_{0}^{\lambda}\left[\tau_{2}-\tau_{1}\right]\right| \geq \varepsilon k \mathbb{E}_{0}^{\lambda}\left[\tau_{2}-\tau_{1}\right]\right] \rightarrow 0 \text { for } k \rightarrow \infty,
$$

uniformly for $\lambda \leq 1$. 
Proof of Lemma 5.13.

We have

$$
\begin{aligned}
& \widehat{\mathbb{P}}_{0}^{\lambda}\left[\left|\frac{\tau_{k}}{k}-\widehat{\mathbb{E}}_{0}^{\lambda}\left[\tau_{2}-\tau_{1}\right]\right| \geq \varepsilon \widehat{\mathbb{E}}_{0}^{\lambda}\left[\tau_{2}-\tau_{1}\right]\right] \\
\leq & \frac{1}{\varepsilon^{2} \widehat{\mathbb{E}}_{0}^{\lambda}\left[\tau_{2}-\tau_{1}\right]^{2}} \widehat{\mathbb{E}}_{0}^{\lambda}\left[\left(\frac{\tau_{k}}{k}-\widehat{\mathbb{E}}_{0}^{\lambda}\left[\tau_{2}-\tau_{1}\right]\right)^{2}\right] \\
\leq & \frac{1}{k^{2} \varepsilon^{2} \widehat{\mathbb{E}}_{0}^{\lambda}\left[\tau_{2}-\tau_{1}\right]^{2}} \widehat{\mathbb{E}}_{0}^{\lambda}\left[\left(\tau_{1}-\widehat{\mathbb{E}}_{0}^{\lambda}\left[\tau_{2}-\tau_{1}\right]+\sum_{j=2}^{k}\left(\tau_{j}-\tau_{j-1}-\widehat{\mathbb{E}}_{0}^{\lambda}\left[\tau_{2}-\tau_{1}\right]\right)\right)^{2}\right] \\
= & \frac{1}{k^{2} \varepsilon^{2} \widehat{\mathbb{E}}_{0}^{\lambda}\left[\tau_{2}-\tau_{1}\right]^{2}}\left(\widehat{\mathbb{E}}_{0}^{\lambda}\left[\left(\tau_{1}-\widehat{\mathbb{E}}_{0}^{\lambda}\left[\tau_{2}-\tau_{1}\right]\right)^{2}\right]+\sum_{j=2}^{k} \widehat{\mathbb{E}}_{0}^{\lambda}\left[\left(\tau_{j}-\tau_{j-1}-\widehat{\mathbb{E}}_{0}^{\lambda}\left[\tau_{2}-\tau_{1}\right]\right)^{2}\right]\right)
\end{aligned}
$$

where we used the independence property stated in Theorem 5.6. But the last term equals

$$
\begin{gathered}
\frac{1}{k^{2} \varepsilon^{2} \widehat{\mathbb{E}}_{0}^{\lambda}\left[\tau_{2}-\tau_{1}\right]^{2}}\left(\widehat{\mathbb{E}}_{0}^{\lambda}\left[\tau_{1}^{2}\right]-2 \widehat{\mathbb{E}}_{0}^{\lambda}\left[\tau_{1}\right] \widehat{\mathbb{E}}_{0}^{\lambda}\left[\tau_{2}-\tau_{1}\right]+\widehat{\mathbb{E}}_{0}^{\lambda}\left[\left(\tau_{2}-\tau_{1}\right)^{2}\right]\right) \\
+\frac{k-1}{k^{2} \varepsilon^{2} \widehat{\mathbb{E}}_{0}^{\lambda}\left[\tau_{2}-\tau_{1}\right]^{2}} \widehat{\mathbb{E}}_{0}^{\lambda}\left[\left(\tau_{2}-\tau_{1}-\widehat{\mathbb{E}}_{0}^{\lambda}\left[\tau_{2}-\tau_{1}\right]\right)^{2}\right]
\end{gathered}
$$

and we conclude, using Lemma 5.10.

Finally, Lemma 5.12 follows from Lemma 5.13 by taking $k=n(t)$ : due to (5.31), $\lim _{\alpha \rightarrow+\infty} \limsup _{\lambda \rightarrow 0 ; t \rightarrow+\infty ; \lambda^{2} t=\alpha} n(t)=\infty$ and

$$
\lim _{\alpha \rightarrow+\infty} \limsup _{\lambda \rightarrow 0 ; t \rightarrow+\infty ; \lambda^{2} t=\alpha} \frac{n(t) \widehat{\mathbb{E}}_{0}^{\lambda}\left[\tau_{2}-\tau_{1}\right]}{t}=1 .
$$

To see that (5.47) holds true, note that

$$
t\left(1-\frac{\lambda^{2} \widehat{\mathbb{E}}_{0}^{\lambda}\left[\tau_{2}-\tau_{1}\right]}{\lambda^{2} t}\right) \leq n(t) \widehat{\mathbb{E}}_{0}^{\lambda}\left[\tau_{2}-\tau_{1}\right] \leq t
$$

and use (5.31).

\section{Proof of the Theorem}

Combine (5.1) with Proposition 3.1.

\section{Extension to measurable coefficients}

Here we explain how the approach developed in the previous Sections of the paper can be extended to deal with measurable coefficients. Thus the assumptions in force in this Section are Assumptions 1, 3 and 4 that remain unchanged and Assumption 2 is replaced by the following weaker statement: 
Assumption 2': for any environment $\omega$, the functions $x \rightarrow V^{\omega}(x)$ and $x \rightarrow \sigma^{\omega}(x)$ are measurable.

In such generality, it is not possible to use stochastic differential equations to define the processes $X^{\omega}$ or $X^{\lambda, \omega}$ anymore so that our first task is to give an alternative construction.

In the following discussion we fix an environment $\omega$ satisfying assumptions 1, 2', 3 and 4 . The case $\lambda=0$ is included.

Let $p^{\lambda, \omega}(t, x, y)$ be the kernel associated to the operator $\mathscr{L}^{\lambda, \omega}$ in equation (2.8), or, in the case $\lambda=0$ to the operator $\mathscr{L}^{\omega}$ in equation (2.1) now understood in weak distributional sense and let $T_{t}^{\lambda, \omega}$ be the corresponding semigroup

$$
T_{t}^{\lambda, \omega} f(x)=\int p^{\lambda, \omega}(t, x, y) f(y) d y .
$$

It follows from Aronson's estimate (see [1]) and Harnack's inequality (see [10]) that $T_{t}^{\lambda, \omega}$ maps continuous functions vanishing at infinity to continuous functions vanishing at infinity. For such functions we also have $\lim _{t \rightarrow 0} T_{t}^{\lambda, \omega} f(x)=f(x)$ for all $x$. Thus $T_{t}^{\lambda, \omega}$ is a Feller semigroup and it follows from [6] Theorem 2.7 that there exists a conservative Hunt process with continuous paths whose semigroup is $T^{\lambda, \omega}$. We denote its law on path space $C\left(\mathbb{R}_{+}, \mathbb{R}^{d}\right)$ with $P_{x}^{\lambda, \omega}$ and $E_{x}^{\lambda, \omega}$ for the corresponding expectation. Observe that $P_{x}^{\lambda, \omega}[X(0)=x]=1$ for all $x$.

It is proved in [20] Proposition 1 that, for almost all $\omega$ 's, under $P_{0}^{\omega}$, the canonical process satisfies an invariance principle with some effective diffusivity matrix $\Sigma$. It follows from Aronson's estimate that $\Sigma$ is also the asymptotic covariance so that Definition 2.1 goes through.

On the other hand all statements in Section 4 as well as Lemma 5.2 in Section 5 were proved under Assumption 2' only. Thus the construction of regeneration times we gave in Section 5 and all the upper bounds on $\tau_{1}$ are still valid with measurable coefficients. Therefore we see that the law of large numbers is satisfied $\mathbb{Q}$ almost surely under $P_{0}^{\lambda, \omega}$ for all positive $\lambda$ and Definition 2.2 can still be used as the definition of the effective drift.

Having defined the effective diffusivity matrix and the effective drift we claim that the Einstein relation stated in Theorem 2.3 holds true with Assumption 2 being replaced by Assumption 2'.

The main difficulties in extending the proofs of the previous sections to measurable coefficients appear in justifying the Girsanov transform and time change arguments from Section 3. Following [20], in order to do it we shall appeal to Dirichlet form theory, as exposed in [9], and related stochastic calculus for Dirichlet processes. Observe that a direct application of Dirichlet form theory a priori only provides information under $P_{x}^{\lambda, \omega}$ for all $\omega$ but only for (Lebesgue) almost all $x$ (in fact for quasi all $x$ but we won't use fine topological notions here) and therefore, as a consequence of the translation invariance of $\mathbb{Q}$, under $P_{0}^{\lambda, \omega}$ for almost all $\omega$. Therefore most claims in Sections 3, such as Lemma 3.3 or formula (3.4), should now be understood 'for $\mathbb{Q}$ almost all $\omega$ 's'. We let the reader convince herself that this does not affect the proofs.

We use the notation $\rho_{\lambda}(x)=e^{\hat{\lambda} \cdot x}$ for $\lambda \geq 0$. Let $L^{2}\left(\rho_{\lambda}^{2}\right)$ be the space of square integrable functions with respect to the measure $\rho_{\lambda}^{2}(x) d x$. Define $H_{1}\left(\rho_{\lambda}^{2}\right)$ to be the space of functions in $L^{2}\left(\rho_{\lambda}^{2}\right)$ whose gradient is also square integrable with respect to the measure $\rho_{\lambda}^{2}(x) d x$. Let

$$
\mathscr{E}^{\lambda, \omega}(f, f):=\frac{1}{2} \int\left|\sigma^{\omega}(x) \nabla f(x)\right|^{2} e^{-2 V^{\omega}(x)} \rho_{\lambda}^{2}(x) d x .
$$

Then $\left(\mathscr{E}^{\lambda, \omega}, H_{1}\left(\rho_{\lambda}^{2}\right)\right)$ is a regular Dirichlet form. We claim that 
Lemma 7.1 $\left(\mathscr{E} \lambda, \omega, H_{1}\left(\rho_{\lambda}^{2}\right)\right)$ is the Dirichlet form of the semigroup $T^{\lambda, \omega}$ on $L^{2}\left(e^{2 \hat{\lambda} \cdot x-2 V^{\omega}(x)} d x\right)$.

(Note that this fact is already used in [20] but without justification.)

Proof

We first observe that $T^{\lambda, \omega}$ is indeed a strongly continuous symmetric semigroup on $L^{2}\left(\rho_{\lambda}^{2}\right)$.

Let $t>0$ and define the approximating bilinear forms

$$
\mathscr{E} t, \lambda, \omega(f, f):=\frac{1}{t} \int\left(f(x)-T_{t}^{\lambda, \omega} f(x)\right) f(x) e^{-2 V^{\omega}(x)} \rho_{\lambda}^{2}(x) d x .
$$

A function $f$ belongs to the domain of the Dirichlet form associated to the semigroup $T^{\lambda, \omega}$ if and only if $\mathscr{E} t, \lambda, \omega(f, f)$ is bounded in $t$ and the limit as $t$ tends to 0 is then the value of the form, see Lemma 1.3.4 in [9].

A straighforward integration by parts shows that, as $t$ tends to 0 , then $\mathscr{E} t, \lambda, \omega(f, f)$ converges to $\mathscr{E}^{\lambda, \omega}(f, f)$ on the $L^{2}$ domain of the generator $\mathscr{L}^{\lambda, \omega}$, say $\mathscr{D}^{\lambda, \omega}$. Since the function $t \rightarrow \mathscr{E}^{t, \lambda, \omega}(f, f)$ is decreasing, it implies that

$$
\mathscr{E}^{t}, \lambda, \omega(f, f) \leq \mathscr{E}^{\lambda, \omega}(f, f)
$$

for $f \in \mathscr{D}^{\lambda, \omega}$. This inequality extends by density to all functions in $H_{1}\left(\rho_{\lambda}^{2}\right)$. Thus we have proved that the Dirichlet form of the semigroup $T^{\lambda, \omega}$ is well defined and coincides with $\mathscr{E} \lambda, \omega$ on $H_{1}\left(\rho_{\lambda}^{2}\right)$ or, in other words, that it is an extension of the form $\left(\mathscr{E} \lambda, \omega, H_{1}\left(\rho_{\lambda}^{2}\right)\right)$. But since $\left(\mathscr{E} \lambda, \omega, H_{1}\left(\rho_{\lambda}^{2}\right)\right)$ is its own maximal Markovian extension, see Theorem 3.3.1 in [9], both forms coincide.

¿From now on we will drop the superscript $\lambda$ from the notation when $\lambda=0$. We now consider properties of the canonical process $X$ for a fixed environment $\omega$ and under $P_{x}^{\omega}$ for almost every starting point $x$. The function $\phi(x)=e_{1} \cdot x$ locally belongs to the domain of the Dirichlet form $\mathscr{E} \omega$. From Theorem 5.5.1 in [9] we deduce that $e_{1} \cdot X(t)-e_{1} \cdot X(0)$ is a local Dirichlet process under $P_{x}^{\omega}$ for almost every starting point $x$. Thus $e_{1} \cdot X(t)-e_{1} \cdot X(0)$ admits a unique Fukushima decomposition as the sum of a local martingale, say $\bar{B}$, and a process of locally vanishing quadratic variation. The bracket of $\bar{B}$ is given by Theorem 5.5.2 in [9] and satisfies

$$
\langle\bar{B}\rangle(t)=\int_{0}^{t}\left|\sigma^{\omega}(X(s)) e_{1}\right|^{2} d s .
$$

By assumption 3, we have $\langle\bar{B}\rangle(t) \leq \kappa^{-1} t$. Thus we see that in fact $\bar{B}$ is a square integrable martingale. By the same argument the exponential local martingale $e^{\mu \bar{B}(t)-\frac{\mu^{2}}{2}\langle\bar{B}\rangle(t)}$ is also seen to be a martingale for all $\mu$ and one proves as in Lemma 3.3 that

$$
E\left[e^{\mu \lambda \bar{B}(t)-\frac{\mu \lambda^{2}}{2}\langle\bar{B}\rangle(t)}\right] \leq e^{(\mu-1) \frac{\mu \lambda^{2}}{2} \frac{t}{\kappa}},
$$

for every $\omega$ and almost all $x$. Note that the translation invariance of $\mathbb{Q}$ then implies that (7.4) also holds for almost all $\omega$ with $x=0$.

We now justify the Girsanov formula:

Proposition 7.2 For any environment $\omega$, for almost any $x$, any $t$ and any continuous bounded function F we have

$$
E_{x}^{\lambda, \omega}[F(X([0, t]))]=E_{x}^{\omega}\left[F(X([0, t])) e^{\lambda \bar{B}(t)-\frac{\lambda^{2}}{2}\langle\bar{B}\rangle(t)}\right] .
$$


The translation invariance of $\mathbb{Q}$ then implies that for almost all environments

$$
E_{0}^{\lambda, \omega}[F(X([0, t]))]=E_{0}^{\omega}\left[F(X([0, t])) e^{\lambda \bar{B}(t)-\frac{\lambda^{2}}{2}\langle\bar{B}\rangle(t)}\right],
$$

for any continuous and bounded function $F$ and we have obtained the almost sure version of the Girsanov formula (3.4) that is sufficient to proceed through the proofs of the previous Sections.

Proof

We would like to invoke Theorem 3.1 of [3] but unfortunately $\phi$ does not belong to $L^{2}$ so that some work is needed.

Let $G_{n}$ be the ball centered at the origin with radius $n$, and let $\zeta_{n}$ be the exit time from $G_{n}$.

We shall first prove that

$$
E_{x}^{\lambda, \omega}\left[F(X([0, t])) ; t<\zeta_{n}\right]=E_{x}^{\omega}\left[F(X([0, t])) e^{\lambda \bar{B}(t)-\frac{\lambda^{2}}{2}\langle\bar{B}\rangle(t)} ; t<\zeta_{n}\right] .
$$

Using the bound (7.4), it is then possible to let $n$ tend to infinity and deduce Proposition 7.2 from (7.5).

Choose a function $\phi_{n}$ that coincides with $\phi$ on $G_{n}$, is smooth and has compact support. Let $M_{n}$ be the martingale part of the process $\phi_{n}(X(t))-\phi_{n}(X(0))$ in its Fukushima decomposition under $P_{x}^{\omega}$, and let $Z_{n}(t):=e^{\lambda M_{n}(t)-\frac{\lambda^{2}}{2}\left\langle M_{n}\right\rangle(t)}$.

Define

$$
Q_{t}^{n, \lambda, \omega} f(x):=E_{x}^{\omega}\left[f(X(t)) Z_{n}(t)\right] .
$$

Then $Q_{t}^{n, \lambda, \omega}$ defines a strongly continuous Markovian semigroup on $L^{2}(d x)$. We need the following

Lemma 7.3 The Dirichlet form of the semigroup $Q_{t}^{n, \lambda, \omega}$ acting on $L^{2}\left(e^{2 \phi_{n}(x)-2 V^{\omega}(x)} d x\right)$ is

$$
\frac{1}{2} \int\left|\sigma^{\omega}(x) \nabla f(x)\right|^{2} e^{2 \phi_{n}(x)-2 V^{\omega}(x)} d x,
$$

with domain $H_{1}(d x)$.

Proof

The Revuz measure of the positive continuous additive functional $\left\langle M_{n}\right\rangle$ is $\left|\nabla \sigma^{\omega}(x) \phi_{n}(x)\right|^{2} d x$ which is easily seen to belong to the Hardy class since the gradient of $\phi_{n}$ is uniformly bounded. Besides condition (3.8) in [3] is fulfilled whenever $\nabla \phi_{n}$ is uniformly bounded. Thus Theorem 3.1 of [3] applies. We get that the quadratic form of the semigroup $Q_{t}^{n, \lambda, \omega}$ acting on $L^{2}\left(e^{-2 V^{\omega}(x)} d x\right)$ is

$$
\begin{aligned}
\mathscr{Q}_{n}(f, g):= & \frac{1}{2} \int \sigma^{\omega}(x) \nabla f(x) \cdot \sigma^{\omega}(x) \nabla g(x) e^{-2 V^{\omega}(x)} d x \\
& -\int g(x) \sigma^{\omega}(x) \nabla f(x) \cdot \sigma^{\omega}(x) \nabla \phi_{n}(x) e^{-2 V^{\omega}(x)} d x,
\end{aligned}
$$

see formula (3.3) in [3].

We now use the same approximating sequence as in (7.2); see also the explanation on page 242 of [3]. We then know that for all functions $f, g \in H_{1}(d x)$

$$
\mathscr{Q}_{n}(f, g)=\lim _{t \rightarrow 0} \frac{1}{t} \int\left(f(x)-Q_{t}^{n, \lambda, \omega} f(x)\right) g(x) e^{-2 V^{\omega}(x)} d x .
$$


Applying this formula to the function $g e^{2 \phi_{n}}$ and using (7.6), we deduce that the Dirichlet form of the semigroup $Q_{t}^{n, \lambda, \omega}$ acting on $L^{2}\left(e^{2 \phi_{n}(x)-2 V^{\omega}(x)} d x\right)$ is

$$
\begin{aligned}
& \lim _{t \rightarrow 0} \frac{1}{t} \int\left(f(x)-Q_{t}^{n, \lambda, \omega} f(x)\right) g(x) e^{2 \phi_{n}(x)} e^{-2 V^{\omega}(x)} d x \\
= & \frac{1}{2} \int \sigma^{\omega}(x) \nabla f(x) \cdot \sigma^{\omega}(x) \nabla\left(g(x) e^{2 \phi_{n}(x)}\right) e^{-2 V^{\omega}(x)} d x \\
- & \int g(x) \sigma^{\omega}(x) \nabla f(x) \cdot \sigma^{\omega}(x) \nabla \phi_{n}(x) e^{2 \phi_{n}(x)-2 V^{\omega}(x)} d x \\
= & \frac{1}{2} \int \sigma^{\omega}(x) \nabla f(x) \cdot \sigma^{\omega}(x) \nabla g(x) e^{2 \phi_{n}(x)-2 V^{\omega}(x)} d x .
\end{aligned}
$$

We can now conclude the proof of Proposition 7.2.

Comparing the expression of the Dirichlet form generated by $Q_{t}^{n, \lambda, \omega}$ we just obtained with formula (7.1) for $\mathscr{E}^{\lambda, \omega}$ and observing that $\phi_{n}=\phi$ on $G_{n}$ we see that the parts of both these Dirichlet forms on $G_{n}$ coincide. Clearly $\bar{B}$ and $M_{n}$ also coincide up to time $\zeta_{n}$. Thus we obtain (7.5).

Finally we should say a word about the time change argument used in the proof of Proposition 3.1 in the case $V \neq 0$. Define $Y^{\lambda, \omega}$ to be the Hunt process with Dirichlet form $\left(\mathscr{E} \lambda, \omega, H_{1}\left(\rho_{\lambda}^{2}\right)\right)$ with reference measure $\rho_{\lambda}^{2}(x) d x$. Theorem 6.2.1 in [9] implies that the process obtained by time changing $Y^{\lambda, \omega}$ through the additive functional $A^{\lambda, \omega}(t)=\int_{0}^{t} e^{-2 V^{\omega}\left(Y^{\lambda, \omega}(s)\right)} d s$ admits as Dirichlet form $\left(\mathscr{E} \lambda, \omega, H_{1}\left(\rho_{\lambda}^{2}\right)\right)$ with reference measure $e^{-2 V^{\omega}(x)} \rho_{\lambda}^{2}(x) d x=e^{2 \hat{\lambda} \cdot x-2 V^{\omega}(x)}$ so that, for almost any initial point $x$, the law of $Y^{\lambda, \omega} \circ\left(A^{\lambda, \omega}\right)^{-1}$ coincides with $P_{x}^{\lambda, \omega}$.

Acknowledgement: we thank J.C. Mourrat for his careful reading of a preliminary version of the paper.

\section{References}

[1] Aronson, D. G. (1968)

Non-negative solutions of linear parabolic equations.

Ann. Scuola Norm. Sup. di Pisa 22(4), 607-694.

[2] Berger, N., Gantert, N., Peres, Y. (2003)

The speed of biased random walk on percolation clusters.

Probab. Theory Related Fields 126, 221-242.

[3] Chen, Z.Q., Fitzsimmons, P.J., Kuwae, K., T.-S. Zhang, T.S. (2008)

Perturbation of symmetric Markov processes.

Probab. Theory Related Fields 140, 239-275.

[4] De Masi, A., Ferrari, P., Goldstein, S., Wick, W.D. (1989)

An invariance principle for reversible Markov processes. Applications to random motions in random environments.

Journ. Stat. Phys. 55 (3/4), 787-855. 
[5] Einstein, A. (1956)

Investigations on the theory of the Brownian movement.

Edited with notes by R. Fürth. Translated by A. D. Cowper. Dover Publications, Inc., New York.

[6] Ethier, S.N., Kurtz T.G. (1986)

Markov processes, characterization and convergence.

Wiley.

[7] Faggionato, A., Mathieu, P. (2008)

Mott law as upper bound for a random walk in a random environment.

Comm. Math. Phys. 281, 263-286.

[8] Faggionato, A., Schulz-Baldes, H., Spehner, D. (2006)

Mott law as lower bound for a random walk in a random environment.

Comm. Math. Phys. 263, 21-64.

[9] Fukushima, M., Oshima, Y., Takeda, M. (1994)

Dirichlet forms and symmetric Markov processes.

de Gruyter Studies in Mathematics, 19. Walter de Gruyter, Berlin.

[10] Gilbarg, D., Trudinger, N.S. (2001)

Elliptic partial differential equations of second order.

Springer-Verlag, Berlin.

[11] Gutierrez, C.E., Wheeden, R.L. (1991)

Harnack's inequality for degenerate parabolic equations. Comm. Partial Differential Equations 16(4-5), 745-770.

[12] Jikov, V.V., Kozlov, S.M., Oleinik, O.A. (1994)

Homogenization of differential operators and integral functionals.

Springer-Verlag, Berlin.

[13] Kipnis, C., Varadhan, S.R.S. (1986)

A central limit theorem for additive functionals of reversible Markov processes and applications to simple exclusions.

Comm. Math. Phys. 104, 1-19.

[14] Kesten, H. (1977)

A renewal theorem for random walk in a random environment.

Symposia Pure. Math. 31, 67-77.

[15] Komorowski, T., Olla, S. (2005)

Einstein relation for random walks in random environments.

Stochastic Process. Appl. 115 (8), 1279-1301.

[16] Komorowski, T., Olla, S. (2005)

On mobility and Einstein relation for tracers random walks in time-mixing random environments.

Journal of Statistical Physics 118 (3-4), 407-435. 
[17] Kozlov, S.M. (1980)

Averaging of Random Operators

Sb. Math. 37 (2), 167-180.

[18] Kozlov, S.M. (1985)

The method of averaging and walks in inhomogeneous environments

Russian Math. Surveys 40 (2), 73-145.

[19] Lebowitz, J.L., Rost, H. (1994)

The Einstein relation for the displacement of a test particle in a random environment.

Stochastic Process. Appl. 54 (2), 183-196.

[20] Lejay, A. (2001)

Homogenization of divergence-form operators with lower-order terms in random media.

Probab. Theory Related Fields 120 (2), 255-276.

[21] Loulakis, M. (2005)

Einstein relation for a tagged particle in simple exclusion processes.

Comm. Math. Phys. 229 (2), 347-367.

[22] Osada, H. (1982)

Homogenization of diffusion processes with random stationary coefficients.

Probability theory and mathematical statistics (Tbilisi, 1982), 507-517, Lecture Notes in Math., 1021, Springer, Berlin, 1983.

[23] Papanicolaou, G., Varadhan, S.R.S. (1982)

Diffusion with random coefficients.

Essays in honor of C.R. Rao, ed. by G. Kallianpur, P.R. Krishnajah, J.K. Gosh pp. 547-552.

Amsterdam: North Holland 1982.

[24] Revuz, D., Yor, M. (1994)

Continuous martingales and Brownian motion.

Grundlehren der Mathematischen Wissenschaften

(Fundamental Principles of Mathematical Sciences) 293. Springer, Berlin.

[25] Shen, L. (2003)

On ballistic diffusions in random environment.

Ann. I. H. Poincaré-PR 39 (5), 839-876.

[26] Sznitman, A. (2003)

On the anisotropic walk on the supercritical percolation cluster.

Comm. Math. Phys. 240, 123-148.

[27] Sznitman, A., Zeitouni, O. (2006)

An invariance principle for isotropic diffusions in random environment.

Invent. Math. 164, 455-567. 
[28] Sznitman, A., Zerner, M. (1999)

A law of large numbers for random walks in random environment. Ann. Probab. 27, 1851-1869. 